\title{
Protoneutron star evolution and the neutrino-driven wind in general relativistic neutrino radiation hydrodynamics simulations
}

\author{
T. Fischer ${ }^{1}$, S. C. Whitehouse ${ }^{1}$, A. Mezzacappa ${ }^{2}$, F.-K. Thielemann ${ }^{1,3}$, and M. Liebendörfer ${ }^{1}$ \\ 1 Department of Physics, University of Basel, Klingelbergstrasse 82, 4056 Basel, Switzerland \\ 2 Physics Division, Oak Ridge National Laboratory, Oak Ridge, Tennessee 37831-1200, USA \\ 3 GSI Helmholtzzentrum für Schwerionenforschung GmbH, Planckstrasse1, 64291 Darmstadt, Germany
}

Received 11 August 2009 / Accepted 28 March 2010

\section{ABSTRACT}

\begin{abstract}
Massive stars end their lives in explosions with kinetic energies on the order of $10^{51} \mathrm{erg}$. Immediately after the explosion has been launched, a region of low density and high entropy forms behind the ejecta, which is continuously subject to neutrino heating. The neutrinos emitted from the remnant at the center, the protoneutron star (PNS), heat the material above the PNS surface. This heat is partly converted into kinetic energy, and the material accelerates to an outflow that is known as the neutrino-driven wind. For the first time we simulate the collapse, bounce, explosion, and the neutrino-driven wind phases consistently over more than 20 s. Our numerical model is based on spherically symmetric general relativistic radiation hydrodynamics using spectral three-flavor Boltzmann neutrino transport. In simulations where no explosions are obtained naturally, we model neutrino-driven explosions for low- and intermediatemass Fe-core progenitor stars by enhancing the charged current reaction rates. In the case of a special progenitor star, the $8.8 \mathrm{M}_{\odot}$ $\mathrm{O}-\mathrm{Ne}-\mathrm{Mg}$-core, the explosion in spherical symmetry was obtained without enhanced opacities. The post-explosion evolution is in qualitative agreement with static steady-state and parametrized dynamic models of the neutrino-driven wind. On the other hand, we generally find lower neutrino luminosities and mean neutrino energies, as well as a different evolutionary behavior of the neutrino luminosities and mean neutrino energies. The neutrino-driven wind is proton-rich for more than $10 \mathrm{~s}$ and the contraction of the PNS differs from the assumptions made for the conditions at the inner boundary in previous neutrino-driven wind studies. Despite the moderately high entropies of about $100 k_{\mathrm{B}}$ /baryon and the fast expansion timescales, the conditions found in our models are unlikely to favor $r$-process nucleosynthesis. The simulations are carried out until the neutrino-driven wind settles down to a quasi-stationary state. About $5 \mathrm{~s}$ after the bounce, the peak temperature inside the PNS already starts to decrease because of the continued deleptonization. This moment determines the beginning of a cooling phase dominated by the emission of neutrinos. We discuss the physical conditions of the quasi-static PNS evolution and take the effects of deleptonization and mass accretion from early fallback into account.
\end{abstract}

Key words. hydrodynamics - neutrinos - radiative transfer - relativistic processes

\section{Introduction}

Stars more massive than $8 \mathrm{M}_{\odot}$ run into gravitational collapse at the end of their evolution, due to pressure loss via the photodisintegration of heavy nuclei and electron captures. The collapse halts at nuclear density, typically $2-4 \times 10^{14} \mathrm{~g} / \mathrm{cm}^{3}$ depending on the equation of state (EoS). However, the supersonically infalling material from the outer core continues to fall into the center. The core overshoots its equilibrium configuration and bounces back. A dynamic shock wave forms, which propagates outwards and continuously loses energy owing to the dissociation of heavy nuclei. As soon as the shock reaches the neutrinospheres, i.e. the neutrino energy and flavor dependent spheres of last scattering, additional electron captures emit a large amount of electron neutrinos. This burst of electron neutrinos, known as the deleptonization burst, carries away energy of several $10^{53} \mathrm{erg} / \mathrm{s}$ on a timescale of $10-20 \mathrm{~ms}$. This energy loss turns the expanding shock into a standing accretion shock (SAS) already about $5 \mathrm{~ms}$ after bounce. Due to the high energy in the neutrino radiation field, neutrino heating between the neutrinospheres and the SAS has long been investigated as a possible source of reviving the SAS and for triggering neutrino-driven explosions (Bethe \& Wilson 1985; Janka 2001; Janka et al. 2005; Mezzacappa et al. 2006).

Up to now, neutrino-driven explosions in spherical symmetry have only been obtained for the low-mass $8.8 \mathrm{M}_{\odot} \mathrm{O}-\mathrm{Ne}-$ Mg-core by Kitaura et al. (2006) and for low- and intermediatemass Fe-core progenitor stars by Sagert et al. (2009) assuming a hadron-quark phase transition during the early post-bounce phase. On the other hand, multi-dimensional core collapse models with spectral neutrino transport have only recently become available. They demonstrate the complexity of the underlying physical phenomena such as rotation and the development of fluid instabilities. Such models have been shown to increase the neutrino heating efficiency (see for example Miller et al. 1993; Herant et al. 1994; Burrows et al. 1995; Janka \& Müller 1996) and help to understand aspherical explosions (see for example Bruenn et al. 2006; Marek \& Janka 2009). For a review of axially-symmetric neutrino-driven explosions, see also Janka et al. (2008).

The following dynamical evolution of the PNS and hence the properties of the neutrino spectra emitted is determined by the mass accretion and the EoS. On a timescale of several seconds after the explosion has been launched, the region between 
the expanding explosion shock, and the PNS at the center is subject to the formation of the neutrino-driven wind as follows. Neutrinos continuously diffuse out of the hot PNS and heat the material on top of the PNS surface before they reach the neutrinospheres. We define the PNS surface to be the radius of the energy-integrated electron-neutrinosphere. The dominant neutrino heating contributions are given by the captures of electronneutrinos and electron-antineutrinos at free nucleons. Matter is heated by neutrinos and the thermal energy is converted into kinetic energy, which accelerates material on top of the PNS surface to positive velocities. This matter outflow is known as the neutrino-driven wind.

In this context, two particular studies are of special importance. The properties of the neutrino-driven wind as described in Woosley et al. (1994) are based on the detailed radiation hydrodynamics simulation of a $20 \mathrm{M}_{\odot}$ Fe-core progenitor applying the numerical model from Wilson \& Mayle (1993). The simulation was carried until about $18 \mathrm{~s}$ after bounce. Another state-of-theart model of that time was the explosion of the O-Ne-Mg-core by Mayle \& Wilson (1988). Both investigations were milestones in the research of core collapse supernovae and are based on detailed neutrino input physics including neutrino transport, developed by J. R. Wilson. The results obtained, in particular the properties of the ejecta and the neutrino observables such as luminosities and energies, were considered the standard reference for more than 10 years. Neutrino-driven wind studies used the results as parameters, where the conditions found indicated the possible site for the production of heavy elements via the $r$-process. In the simulations discussed in the present paper, we follow a similar approach as Woosley et al. (1994) and Mayle \& Wilson (1988), where we apply the neutrino input physics based on Bruenn (1985). Although the previous work is in qualitative agreement with our findings, in particular the explosion phase, significant differences occur in several properties of the neutrino-driven wind. The entropies per baryon are lower by a factor of 2-3 and the wind stays proton-rich for more than $10 \mathrm{~s}$ for all our models. In addition, the neutrino luminosities and mean energies are generally lower. The mean neutrino energies decrease with time, where they remain almost constant in the simulation of Woosley et al. (1994). The largest difference arises in the decreasing difference between the mean electron neutrino and antineutrino energies found in our simulations, i.e. the neutrino spectra become more similar with respect to time. The difference in the neutrino spectra in Woosley et al. (1994) remains large and even increases with time. Using the results from Woosley et al. (1994) as reference, Qian \& Woosley (1996) analyzed the neutrino-driven wind and formulated approximate analytical expressions for various properties of the neutrino-driven wind, e.g. the neutrino heating rate, the electron fraction, the entropy per baryon and the mass outflow rate.

Based on the static wind equations, the results obtained in parameter studies (see e.g. Duncan et al. 1986; Hoffman et al. 1997; Thompson et al. 2001; Thompson \& Burrows 2001) became known as static steady-state wind models, where Woosley \& Baron (1992), Woosley et al. (1994), Takahashi et al. (1994) and Witti et al. (1994) described the neutrino-driven wind in a radiation-hydrodynamics context. Of special importance for the neutrino-driven wind investigations is the impact to the nucleosynthesis. Most interesting is the possibility to explain the production of heavy elements via the $r$-process due to the high entropies per baryon, the fast expansion timescales and the low electron fraction of $Y_{\mathrm{e}}<0.5$ in the wind. Otsuki et al. (2000) explored general relativistic effects of the neutrino-driven wind and investigated the possible impact to the nucleosynthesis.
Recently, Wanajo (2006a) and Wanajo (2006b) investigated the neutrino-driven wind with respect to the $r$ - and $r p$-processes.

The possibility of supersonic wind velocities has been explored in most of the references. The supersonically expanding material in the wind collides with the much slower expanding and denser explosion ejecta. The material decelerates and a reverse shock forms which is known as the neutrino-driven wind termination shock (first observed by Janka \& Müller 1995; Burrows et al. 1995). Recently, Arcones et al. (2007) examined the post-bounce phase of core collapse supernovae of several massive progenitor stars. Their models were launched from massive progenitor stars that were previously evolved through the core collapse, bounce and early post-bounce phases using sophisticated radiation hydrodynamics based on spectral neutrino transport in spherical symmetry. The simulations were then continued applying a simplified radiation hydrodynamics description (see Scheck et al. 2006), assuming high luminosities to trigger neutrino-driven explosions in spherical symmetry. The neutrino-driven wind develops supersonic outflow and the wind termination shock appears in all their models. Like most of the present neutrino-driven wind studies, an interior boundary was assumed instead of simulating the PNS interior for the PNS contraction and the diffusion of neutrinos out of the PNS. However, steady-state wind studies could not predict the important dynamical features from the presence of the neutrino-driven wind termination shock, especially the deceleration of the wind material and the consequent entropy as well as density and temperature increase during the deceleration. In this respect, the investigation from Arcones et al. (2007) was a milestone in modeling the neutrino-driven wind consistently. On the other hand, they were focusing on parameters (luminosities and mean neutrino energies) in agreement with the simulations of Bethe \& Wilson (1985) and Woosley et al. (1994). Although the dynamics is in general agreement, several properties of the neutrino-driven wind as well as the neutrino spectra emitted differ significantly from our findings.

The present paper follows a different approach. We simulate consistently the dynamical evolution of the collapse, bounce and post-bounce phases until the neutrino-driven wind phase for more than $20 \mathrm{~s}$. The simulations are launched from the $8.8 \mathrm{M}_{\odot}$ O-Ne-Mg-core from Nomoto $(1983,1984,1987)$ and the 10.8 and $18 \mathrm{M}_{\odot}$ Fe-core progenitors from Woosley et al. (2002). Our numerical model is based on general relativistic radiation hydrodynamics with spectral three-flavor Boltzmann neutrino transport in spherical symmetry. The explosion mechanism of massive $\mathrm{Fe}$-core progenitors is an active subject of research. To model neutrino-driven explosions for such progenitors in spherical symmetry, we enhance the electronic charged current reaction rates artificially which increases the neutrino energy deposition and revives the SAS. The mechanism including the tuned neutrino reaction rates will be further discussed in Sects. 2 and 3. Such explosion models were investigated with respect to the nucleosynthesis by Fröhlich et al. (2006a-c). Here, we report on the formation of the neutrino-driven wind and the possibility of the wind developing supersonic velocities and hence the formation of the wind termination shock. In addition, we will also illustrate the explosion and the neutrino-driven wind for the O-Ne-Mg core, where the explosion is obtained in spherical symmetry applying the standard neutrino opacities. The results are in qualitative agreement with those of Mayle \& Wilson (1988) and Kitaura et al. (2006), who used a different EoS.

We find that for low progenitor masses, the neutrinodriven wind termination shock will develop, using the tuned neutrino reaction rates. When the neutrino reaction rates are 
switched back to the standard opacities given in Bruenn (1985), the neutrino-driven wind develops only a subsonic matter outflow. For intermediate progenitor masses, the neutrino-driven wind remains subsonic even with the artificially enhanced neutrino emission and absorption rates. Since the neutrino-driven wind depends sensitively on the emitted neutrino spectra at the neutrinospheres, we believe accurate neutrino transport and general relativity in the presence of strong gravitational fields are essential in order to describe the dynamical evolution. Furthermore, the accurate modeling of the electron fraction in the wind is essential for nucleosynthesis calculations, which can only be obtained solving the neutrino transport equation. In addition, it is beyond the present computational capabilities to carry multi-dimensional simulations with neutrino transport to several seconds after bounce. Hence, our investigations are performed in spherical symmetry where we simulate the entire PNS interior rather than approximating an interior boundary. We find significant discrepancies in comparison with the assumptions made in previous wind studies. Material is found to be proton-rich for more than $10 \mathrm{~s}$, where most wind models assume luminosities and mean neutrino energies such that the neutrino-driven wind becomes neutron-rich. We question the validity of the approximations made in such wind studies. We believe that the accurate and consistent modeling of the physical conditions in the neutrino-driven wind is essential, especially in order to be able to draw conclusions with respect to the nucleosynthesis.

The paper is organized as follows. In Sect. 2, we will present our spherically symmetric core collapse model. Section 3 is devoted to the explosion phase of neutrino-driven explosions in spherical symmetry. We examine the $8.8 \mathrm{M}_{\odot}$ progenitor model from Nomoto $(1983,1984,1987)$ using the standard neutrino opacities and the 10.8 and $18 \mathrm{M}_{\odot}$ progenitor models from Woosley et al. (2002) using artificially enhanced neutrino reaction rates. In Sect. 4 we discuss the conditions for the formation of the neutrino-driven wind and the possibility for the wind to develop supersonic velocities. We discuss in Sect. 5 the electron fraction approximation used in the literature. Since a generally neutron-rich neutrino-driven wind is found in many previous and present wind studies, we illustrate the differences and investigate why we find a generally proton-rich wind. Section 6 is dedicated to the long term post-bounce evolution for more than $20 \mathrm{~s}$. In Sect. 7 we discuss the results and emphasize the main differences of the present investigation to previous wind studies. Finally we close with a summary in Sect. 8 .

\section{The model}

Our core collapse model, AGILE-BOLTZTRAN, is based on general relativistic radiation hydrodynamics in spherical symmetry, using three-flavor (anti)neutrino Boltzmann transport. For details see Mezzacappa \& Bruenn (1993a-c), Mezzacappa \& Messer (1999), Liebendörfer et al. (2001a,b) and Liebendörfer et al. (2004). For this study we include the neutrino input physics based on Bruenn (1985). The charged current reactions considered

$\mathrm{e}^{-}+\mathrm{p} \rightleftharpoons \mathrm{n}+v_{\mathrm{e}}$

$\mathrm{e}^{+}+\mathrm{n} \rightleftharpoons \mathrm{p}+\bar{v}_{\mathrm{e}}$

$\mathrm{e}^{-}+\langle A, Z\rangle \rightleftharpoons\langle A, Z-1\rangle+v_{\mathrm{e}}$

are electron and positron captures at free nucleons as well as electron captures at nuclei. The nuclei are characterized by an average atomic mass and charge $\langle A, Z\rangle$. In addition, the standard scattering reactions considered are iso-energetic neutrino nucleon $(N \in\{n, p\})$ and nuclei $(N=\langle A, Z\rangle)$ scattering,

$v+N \rightleftharpoons v+N$,

where $v \in\left\{v_{\mathrm{e}}, v_{\mu / \tau}\right\}$ (equivalent for antineutrinos $\bar{v}$ ), and neutrino electron/positron scattering

$v+\mathrm{e}^{ \pm} \rightleftharpoons v+\mathrm{e}^{ \pm}$.

The classical neutrino pair process is electron-positron annihilation,

$\mathrm{e}^{-}+\mathrm{e}^{-} \rightleftharpoons v+\bar{v}$

The standard neutrino energy $E$ dependent emissivity $j(E)$ and absorptivity $\chi(E)$ for the charged current reactions as well as the scattering and pair-reaction rates are given in Bruenn (1985) based on Yueh \& Buchler (1976) and Schinder \& Shapiro (1982). The additional pair-process nucleon-nucleon-Bremsstrahlung,

$N+N \rightleftharpoons N+N+v+\bar{v}$,

has been implemented into our model according to Thompson \& Burrows (2001) and is also taken into account. The emission of $(\mu / \tau)$-neutrino pairs via the annihilation of trapped electronneutrino pairs,

$v_{\mathrm{e}}+\bar{v}_{\mathrm{e}} \rightleftharpoons v_{\mu / \tau}+\bar{v}_{v / \tau}$

as well as contributions from nucleon-recoil and weak magnetism as studied in Horowitz (2002) are investigated in Fischer et al. (2009) and are not taken into account in the present study of the neutrino-driven wind.

\subsection{Recent improvements of the adaptive grid}

Long-term simulations of the supernova post-bounce phase with AGILE-BOLTZTRAN lead to a very large contrast of densities, reaching from $\sim 10^{15} \mathrm{~g} / \mathrm{cm}^{3}$ at the center of the protoneutron star (PNS) to densities on the order of $\mathrm{g} / \mathrm{cm}^{3}$ and lower in the outer layers. The version of AGILE described in Liebendörfer et al. (2002) is not able to resolve such large density contrasts. If the enclosed mass $a$ is large and the density in one zone very low, then the evaluation of the mass contained in the zone according to Eq. (39) in Liebendörfer et al. (2002),

$\mathrm{d} a_{i+\frac{1}{2}}=a_{i+1}-a_{i}$,

is subject to large cancellation so that truncation errors inhibit the convergence of the Newton-Raphson scheme in the implicitly finite differenced time step. However, the problem can be avoided by a simple modification that was first explored in Fisker (2004, priv. comm.). The state vector of AGILE-BOLTZTRAN is given by the following set of quantities

$y=\left(a, r, u, m, \rho, T, Y_{\mathrm{e}}\right)$,

with enclosed baron mass $a$, radius $r$, velocity $u$, gravitational mass $m$, baryon density $\rho$, temperature $T$, electron fraction $Y_{\mathrm{e}}$. In the improved version, the state vector at time $t^{n}$ is based on zone masses, $\mathrm{d} a_{i+\frac{1}{2}}^{n}$, where the enclosed mass

$a_{i}^{n}=\sum_{1}^{i-1} \mathrm{~d} a_{i+\frac{1}{2}}^{n}$

becomes the derived quantity. 
The form of the generic Eq. (30) in Liebendörfer et al. (2002) applies to the continuity equation, the momentum equation and the energy equation. If we define $\delta_{i}=a_{i}^{n+1}-a_{i}^{n}$ as the difference of the enclosed mass $a_{i}$ between time $t^{n}$ and $t^{n+1}$, Eq. (30) in Liebendörfer et al. (2002) becomes

$\frac{y_{i+\frac{1}{2}}^{n+1}\left(\mathrm{~d} a_{i+\frac{1}{2}}^{n}+\delta_{i+1}-\delta_{i}\right)-y_{i+\frac{1}{2}}^{n} \mathrm{~d} a_{i+\frac{1}{2}}^{n}}{\mathrm{~d} t}=y_{i+1}^{\mathrm{adv}}-y_{i}^{\mathrm{adv}}-y_{i+\frac{1}{2}}^{\mathrm{ext}}=0,(5)$

where the relative velocity between the fluid and the grid in the advection term $y^{\text {adv }}$ is defined by

$u_{i}^{\mathrm{rel}}=-\frac{a_{i}^{n+1}-a_{i}^{n}}{\mathrm{~d} t}=-\frac{\delta_{i}}{\mathrm{~d} t}$.

The cancellation of large numbers during the Newton-Raphson iterations of the implicit time step is avoided if the time shifts $\delta_{i}$ are chosen as the unknowns in the state vector when Eqs. (5) and (6) are solved. The vector of zone masses is then updated between the implicit time steps by

$\mathrm{d} a_{i+\frac{1}{2}}^{n+1}=\mathrm{d} a_{i+\frac{1}{2}}^{n}+\delta_{i+1}-\delta_{i}$.

This leads to satisfactory convergence of the Newton-Raphson iterations even in the presence of large density contrasts.

\subsection{The equation of state}

For the present investigation of the neutrino-driven wind, the baryon EoS from Shen et al. (1998) for hot and dense nuclear matter has been implemented for matter in nuclear statistical equilibrium (NSE). For temperatures below $T=0.5 \mathrm{MeV}$ where NSE does not apply, the baryon EoS combines an ideal gas approximation for a distribution of nuclei, based on Timmes \& Arnett (1999) (including ion-ion-correlations), and a nuclear reaction network using the composition given by the progenitor model. Details of the reaction network are given in Thielemann et al. (2004) and references therein. The nuclear abundances are included in the state vector of AGILE-BOLTZTRAN, which reads as follows

$y=\left(a, r, u, m, \rho, T, Y_{\mathrm{e}}, Y_{1}, \ldots, Y_{N}\right)$.

For all these quantities, including the nuclear abundances $Y_{1}, \ldots, Y_{N}$, the corresponding advection equations are solved as described in Liebendörfer et al. (2002) Sect. 3, but with an improved second order accurate total variation diminishing advection scheme. The nuclear reaction network is used in an operator-split manner in order to calculate the abundance changes due to the source terms which in turn depend on employed reaction rates.

Due to computational limitations, we restrict ourselves to $N=19$. We consider the free nucleons and the 14 symmetric nuclei, from ${ }^{4} \mathrm{He}$ to ${ }^{56} \mathrm{Ni}$. In order to model matter with $Y_{\mathrm{e}} \neq 0.5$ to some extent, we additionally include ${ }^{53} \mathrm{Fe},{ }^{54} \mathrm{Fe}$ and ${ }^{56} \mathrm{Fe}$. The network calculates the composition dynamically from the progenitor stage until the simulations are stopped. It is used for an accurate internal energy evolution. In addition, we can approximately reflect the composition of the PNS surface for more than $20 \mathrm{~s}$ after bounce, where nuclei that have been previously in NSE are freezing out of NSE as the temperature drops rapidly below $0.5 \mathrm{MeV}$ already about $1 \mathrm{~s}$ after bounce and reach below $0.01 \mathrm{MeV}$ at about $10 \mathrm{~s}$ post-bounce. In previous studies the simplification of an ideal gas of Si-nuclei was used for matter which is not in NSE. This leads to an increasing inaccurate internal energy evolution after $500 \mathrm{~ms}$ post-bounce when the explosion shock reaches the Si-layer of the progenitor and simplifications could not be extended beyond $1 \mathrm{~s}$ post-bounce time. The implementation of the nuclear reaction network now makes it possible to include more mass (up to and including a large fraction of the He-layer, depending on the progenitor model) into the physical domain and follow the dynamical evolution of the explosion by one order of magnitude longer.

The baryon EoSs for NSE and for non-NSE are coupled to an electron-positron EoS (including photons), developed by Timmes \& Arnett (1999).

\subsection{Enhanced neutrino emissivity and opacity}

By our choice of a spherically symmetric approach, we implement the explosion mechanism of massive Fe-core progenitor stars artificially to trigger neutrino-driven explosions during the post-bounce evolution after the deleptonization burst has been launched. Neutrino heating between the neutrinospheres and the SAS transfers energy from the radiation field into the fluid. A part of this energy is converted into thermal energy which revives the SAS and launches the explosion. The revival of the SAS and hence the neutrino-driven explosions take place on a timescale of several $100 \mathrm{~ms}$.

During the post-bounce evolution, heavy nuclei continue to fall onto the SAS and dissociate into free nucleons. These free nucleons accrete onto the PNS surface. Hence the dominant neutrino heating contributions behind the SAS are due to the electronic charged current reactions, expressions (1) and (2). To trigger explosions in spherically symmetric core collapse simulations of massive Fe-core progenitors, we enhance the emissivity $j$ and absorptivity $\chi$ by a certain factor (typically $5-7$ ) in the region between the SAS and the neutrinospheres. This corresponds to matter with entropies above $6 k_{\mathrm{B}}$ /baryon and baryon densities below $10^{10} \mathrm{~g} / \mathrm{cm}^{3}$. The entropies ahead of the shock are lower and the central densities of the PNS are higher, such that the artificial heating only applies to the region between the neutrinospheres and the SAS. The artificially enhanced reaction rates do not change the neutrino luminosities and mean neutrino energies significantly for the electron-neutrinos and electronantineutrinos. Furthermore, $\beta$-equilibrium is fulfilled since the reverse reaction rates are obtained via the detailed balance. However, the timescale for weak-equilibrium to be established is reduced and hence the electron fraction changes on a shorter timescale. In combination with the increased neutrino energy deposition, this leads to a deviation of the thermodynamic variables in comparison to simulations using the standard opacities given in Bruenn (1985), which will be further discussed in Sect. 7. The weak neutrino-driven explosions obtained have explosion energies of $6.5 \times 10^{50} \mathrm{erg}$ and $2 \times 10^{50} \mathrm{erg}$ for the 10.8 and the $18 \mathrm{M}_{\odot}$ progenitor model respectively.

\subsection{Explosion energy and mass cut}

The explosion energy estimate is a quantity which changes during the dynamical evolution of the system. It is given by the total specific energy of the fluid in the laboratory frame

$E_{\text {specific }}(t, a)=\Gamma e+\frac{2}{\Gamma+1}\left(\frac{u^{2}}{2}-\frac{m}{r}\right)$ 
which in turn is the sum of the specific internal energy $e^{1}$, the specific kinetic energy given by the fluid velocity $u=\partial r / \alpha \partial t$ squared and the specific gravitational binding energy $m / r$ with gravitational mass $m$ and radius $r$ (see Liebendörfer et al. 2001b). $\Gamma(t, a)=\sqrt{1-2 m / r+u^{2}}$ and $\alpha(t, a)$ are the metric functions in a non-stationary and spherically symmetric spacetime with coordinate time $t$, baryon mass $a$ and the two angular coordinates $(\theta, \phi)$ describing a 2 -sphere of radius $r(t, a)$ (see Misner $\&$ Sharp 1964). The explosion is determined by the energy of the ejecta. Integrating $E_{\text {specific }}(t, a)$ with respect to the enclosed baryon mass starting from the progenitor surface $M$ toward the center

$E_{\text {total }}\left(t_{0}, a_{0}\right)=-\int_{M}^{a_{0}} E_{\text {specific }}\left(t_{0}, a\right) \mathrm{d} a$,

gives the total mass-integrated energy, at a given time $t_{0}$ outside a given mass $a_{0}$. The expression (9) is negative during the collapse, bounce and the early post-bounce phases because the progenitor and central Fe-core are gravitationally bound. At some time after bounce, expression (9) becomes positive in the region between the shock and the neutrinospheres. It stays negative at large distances and close to the deep gravitational potential of the PNS, because the outer layers of the progenitor and the PNS continue to be gravitationally bound. While the emission of neutrinos cools the PNS interior, neutrino absorption deposits energy on the timescale $\tau_{\text {heating }}$ on the order of $100 \mathrm{~ms}$ into the fluid near the neutrinospheres. This increases the specific internal energy which matches at later $(\sim 500 \mathrm{~ms})$ post-bounce times the gravitational binding energy at a certain distance toward the center.

On a suggestion by $\mathrm{S}$. Bruenn, we consider the mass cut as follows

$a_{\text {cut }}=a\left(\max \left(E_{\text {total }}\left(t \gg \tau_{\text {heating }}, a\right)\right)\right)$.

The material outside of $a_{\text {cut }}$ is gravitationally unbound and will be ejected while the enclosed material will accrete onto the central PNS. The explosion energy estimate is defined as the total mass-integrated energy of the layers outside the mass cut

$E_{\text {expl }}=E_{\text {total }}\left(t \gg \tau_{\text {heating }}, a_{\text {cut }}\right)$,

at late times ( $t \gg \tau_{\text {heating }}$ ) after the explosion has been launched. It becomes clear from the above expressions that the explosion energy estimate is sensitively determined by the balance of internal and kinetic energies to gravitational binding energy.

From the time post-bounce when the shock reaches low enough densities and temperatures such that neutrinos decouple from matter completely, neutrino heating and cooling does not affect the explosion energy estimate anymore. The additional energy deposition from the neutrino-driven wind, which will be discussed further below, might affect the explosion estimate at later times. We will illustrate in particular the effect of the formation of a supersonic neutrino-driven wind and the wind termination shock to the explosion energy estimate. Only after the neutrino-driven wind disappears, the final value of the explosion energy can be obtained.

1 The baryon contribution to the internal energy is composed of a thermal and nuclear part, i.e. $e=e_{\text {thermal }}+e_{\text {nuclear }}$. In NSE, $e$ is given implicitly via the EoS of hot and dense nuclear matter. In non-NSE, $e_{\text {nuclear }}$ is the binding energy of the nuclear composition used in the reaction network.

\subsection{The neutrino observables}

The neutrino radiation hydrodynamics equations are a coupled system which combines the evolution of the matter properties given by the state vector $y$ and the radiation field, as documented in Liebendörfer et al. (2004) and references therein. The neutrino radiation field is taken into account via the phasespace distribution function $f_{v}(t, a, \mu, E)$ for each neutrino flavor $v=\left(v_{\mathrm{e}}, \bar{v}_{\mathrm{e}}, v_{\mu / \tau}, \bar{v}_{\mu / \tau}\right)$. In spherical symmetry, it depends on the time $t$, the enclosed baryon mass $a$ as well as on the neutrino energy $E$ and the cosine of the propagation angle $\mu=\cos (\theta)$. The evolution of the neutrino radiation field is taken into account by solving the Boltztran transport equation for massless fermions. It determines the phase-space derivative of the specific distribution function $F_{v}=f_{v} / \rho$, i.e. the distribution function divided by the matter density $\rho$, in a co-moving frame (see for example Eq. (8) of Liebendörfer et al. 2005) and due to neutrino-matter interactions such as emission and absorption as well as scattering and pair reactions.

In order to compare simulation results, neutrino observables can be defined. Commonly used are the neutrino numberluminosities, which is given by the phase-space integration of the neutrino distribution function as follows

$L_{n}\left(\left[t_{0}, t_{1}\right], a\right)=4 \pi r^{2} \rho \frac{2 \pi c}{(h c)^{3}} \int_{-1}^{+1} \mathrm{~d} \mu \int_{0}^{\infty} E^{2} \mathrm{~d} E F_{v}(t, a, \mu, E)$,

which is the number of neutrinos ${ }^{2}$ of energy $E$ passing through the mass coordinate $a$ for a given time-interval $\left[t_{0}, t_{1}\right]$ taken in a co-moving frame at position $r(t, a)$. Equivalently, the energyluminosity can be defined as follows

$$
\begin{aligned}
L_{\mathrm{e}}\left(\left[t_{0}, t_{1}\right], a\right) & \equiv L_{v}\left(\left[t_{0}, t_{1}\right], a\right) \\
& =4 \pi r^{2} \rho \frac{2 \pi c}{(h c)^{3}} \int_{-1}^{+1} \mu \mathrm{d} \mu \int_{0}^{\infty} E^{3} \mathrm{~d} E F_{v}(t, a, \mu, E),
\end{aligned}
$$

for each neutrino flavor, i.e. $\left(v_{\mathrm{e}}, \bar{v}_{\mathrm{e}}, v_{\mu / \tau}, \bar{v}_{\mu / \tau}\right)$. Additionally useful quantities are the mean neutrino and root-meansquared (rms) neutrino energies, defined as follows

$$
\begin{aligned}
& \left\langle E_{v}(t, a)\right\rangle=\frac{\int_{-1}^{+1} \mathrm{~d} \mu \int_{0}^{\infty} E^{3} \mathrm{~d} E F_{v}(t, a, \mu, E)}{\int_{-1}^{+1} \mathrm{~d} \mu \int_{0}^{\infty} E^{2} \mathrm{~d} E F_{v}(t, a, \mu, E)}, \\
& \left\langle E_{v}(t, a)\right\rangle_{\mathrm{rms}}=\sqrt{\frac{\int_{-1}^{+1} \mathrm{~d} \mu \int_{0}^{\infty} E^{4} \mathrm{~d} E F_{v}(t, a, \mu, E)}{\int_{-1}^{+1} \mathrm{~d} \mu \int_{0}^{\infty} E^{2} \mathrm{~d} E F_{v}(t, a, \mu, E)}} .
\end{aligned}
$$

We will use these observables, i.e. the energy-luminosities and the mean and root-mean-squared energies, to illustrate the dynamical evolution of the radiation field as well as for comparisons with previous studies.

\subsection{The electron fraction}

The proton-to-baryon ratio is essential for the composition of the ejecta, which is obtained via detailed post processing nucleosynthesis calculations. In the absence of muons or tauons, the proton-to-baryon ratio is given by the electron fraction as follows

$Y_{\mathrm{e}}=Y_{\mathrm{e}^{-}}-Y_{\mathrm{e}^{+}}=Y_{\mathrm{p}}$,

\footnotetext{
2 The integration with respect to $\mu$ is performed separately for in- and out-ward direction, according to the transport coefficients.
} 
which is equal to the number of protons and defines the total number of charges per baryon. The change of the electron fraction is given by the balance of emitted and absorbed electrons (positrons) and electron-neutrinos (antineutrinos) at free nucleons and nuclei. Weak-equilibrium is achieved if

$\mu_{\mathrm{e}^{-}}+\mu_{\mathrm{p}}=\mu_{\mathrm{n}}+\mu_{v_{\mathrm{e}}}$,

$\mu_{\mathrm{e}^{+}}+\mu_{\mathrm{n}}=\mu_{\mathrm{p}}+\mu_{\bar{v}_{\mathrm{e}}}$,

where $\mu_{i}$ are the chemical potentials for electron and positron $\left(\mu_{\mathrm{e}^{ \pm}}\right)$, proton $\left(\mu_{\mathrm{p}}\right)$, neutron $\left(\mu_{\mathrm{n}}\right)$ and electronneutrino $\left(\mu_{v_{\mathrm{e}}}\right)$ and electron-antineutrino $\left(\mu_{\bar{v}_{\mathrm{e}}}\right)$. The timederivative of the electron fraction, $\dot{Y}_{\mathrm{e}}$, is given by the phase-space integration of the emissivities $j_{v}$ and the absorptivities $\chi_{v}$ for electron-neutrinos and electron-antineutrinos as follows

$$
\begin{aligned}
\dot{Y}_{\mathrm{e}}= & -\frac{2 \pi}{(h c)^{3}} \frac{m_{\mathrm{B}} c}{\rho} \int_{-1}^{+1} \mathrm{~d} \mu \int_{0}^{\infty} E^{2} \mathrm{~d} E \\
& \times\left(\left(j_{v_{\mathrm{e}}}-\tilde{\chi}_{v_{\mathrm{e}}} f_{v_{\mathrm{e}}}\right)-\left(j_{\overline{v_{\mathrm{e}}}}-\tilde{\chi}_{\overline{v_{\mathrm{e}}}} f_{\overline{v_{\mathrm{e}}}}\right)\right)
\end{aligned}
$$

where $m_{\mathrm{B}}$ is the baryon mass, $\rho$ is the matter density and $\tilde{\chi}=j+\chi$. The emissivities $j_{v}(E)$ and absorptivities $\chi_{v}(E)$ depend on the neutrino energy. They are the reaction rates for the electronic charged current reactions, which are calculated following Bruenn (1985) and depend on temperature and density. Equation (15) is found by combining the equation of lepton number conservation with the phase-space integrated Boltzmann transport equation (see Mezzacappa \& Bruenn 1993a). To follow the dynamical evolution of the electron fraction via Eq. (15), knowledge of the neutrino distribution functions is required for which neutrino transport is necessary.

\section{Explosions in spherical symmetry}

Progenitor stars more massive than $9 \mathbf{M}_{\odot}$ develop extended $\mathrm{Fe}$-cores at the end of stellar evolution. The explosion mechanism of such Fe-core progenitors is an active subject of research. In the following section, we will investigate the neutrino-driven explosions of the 10.8 and $18 \mathrm{M}_{\odot} \mathrm{Fe}$-core progenitors from Woosley et al. (2002) in spherical symmetry by enhancing the electronic charged current reaction rates artificially. Further below, we will investigate the explosion phase of the $8.8 \mathrm{M}_{\odot} \mathrm{O}-\mathrm{Ne}-$ Mg-core from Nomoto $(1983,1984,1987)$, where the explosion is obtained using the standard neutrino opacities as introduced in Sect. 2.

\subsection{Neutrino-driven explosions of Fe-core progenitors}

The dynamical behavior of the explosion energy estimate and the shock position are illustrated in Figs. 1a and $\mathrm{b}$ respectively with respect to time after bounce. The figures illustrate the explosion phase and the long term evolution up to $10 \mathrm{~s}$ after bounce. After achieving a convergent value between $600 \mathrm{~ms}$ and $2 \mathrm{~s}$ post-bounce of $4.5 \times 10^{50} \mathrm{erg}$, the explosion energy estimate is lifted slightly to about $6.5 \times 10^{50} \mathrm{erg}$. This effect coincides with the additional mass outflow obtained in the neutrinodriven wind and the appearance of the reverse shock, which will be discussed further below. In simulations with a less intense (subsonic) neutrino-driven wind, this effect is negligible and the explosion energy can be obtained already after about $1 \mathrm{~s}$ post-bounce.

The neutrino luminosities and the mean as well as rms neutrino energies are shown in Fig. 2 for the 10.8 (middle panel) and

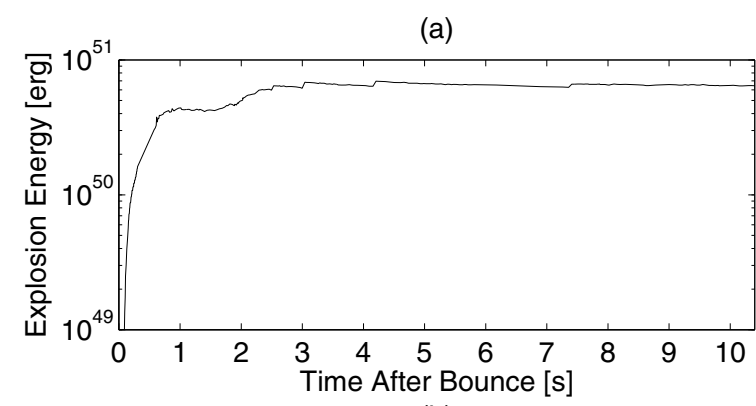

(b)

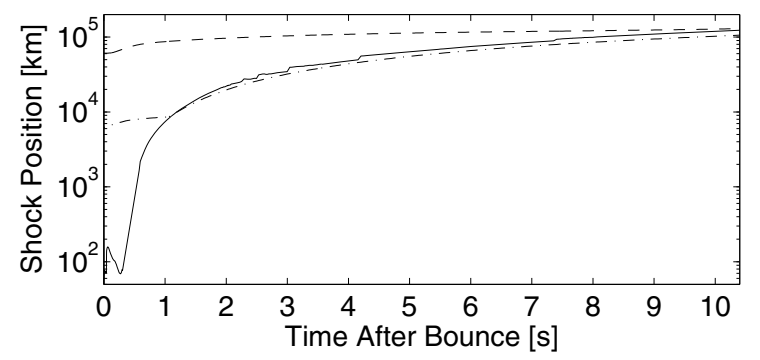

Fig. 1. Explosion energy estimate and shock position with respect to time after bounce for the $10.8 \mathrm{M}_{\odot}$ progenitor model from Woosley et al. (2002). In addition, graph b) illustrates the position of the He-layer (dashed line) and the O-layer (dash-dotted line).

the 18 (right panel) $\mathrm{M}_{\odot}$ progenitor model with respect to time after bounce. Note that the more compact PNS of the $18 \mathrm{M}_{\odot}$ progenitor model results in generally higher neutrino luminosities. The oscillating shock position and the consequent contracting and expanding neutrinospheres during the neutrino heating phase of the 10.8 and $18 \mathrm{M}_{\odot}$ progenitor models on a timescale of several $100 \mathrm{~ms}$ are reflected in the electron-flavor neutrino luminosities, which correspondingly increase and decrease respectively. During the heating phase, the mean neutrino energies of the electron-(anti)neutrinos increase from about 8 (10) MeV after bounce to about 12 (14) MeV until the explosion is launched. The mean neutrino energy of the $(\mu / \tau)$-neutrinos remains constant at about $18 \mathrm{MeV}$ during the heating phase. The mean neutrino energies are generally lower than the rms-energies but follow the same behavior. The explosions for both models are launched after about $350 \mathrm{~ms}$ post-bounce, which defines the neutrino heating timescale for the energy deposition in the gain region to revive the SAS. Matter is accelerated to positive velocities and the SAS turns into the dynamic explosion shock. The resulting neutrino spectra from these artificially induced explosions in spherical symmetry are in general agreement with the neutrino spectra from axially-symmetric neutrino-driven core collapse supernova models that explode without artificially modified reaction rates (see Marek \& Janka 2009). The explosion shock continuously propagates through the remaining domain of the progenitor star. After the explosions have been launched, the electron flavor neutrino luminosities decay exponentially. Furthermore the jumps in the neutrino energies after $350 \mathrm{~ms}$ post-bounce for the 10.8 and $18 \mathrm{M}_{\odot}$ progenitor models are due to the shock propagation over the position of $500 \mathrm{~km}$, where the observables are measured in a co-moving reference frame.

\subsection{The O-Ne-Mg-core}

A special star is the $8.8 \mathrm{M}_{\odot}$ progenitor model from Nomoto (1983, 1984, 1987). The central thermodynamic conditions at the end of stellar evolution are such that only a tiny fraction of 
T. Fischer et al.: protoneutron star evolution and the neutrino-driven wind
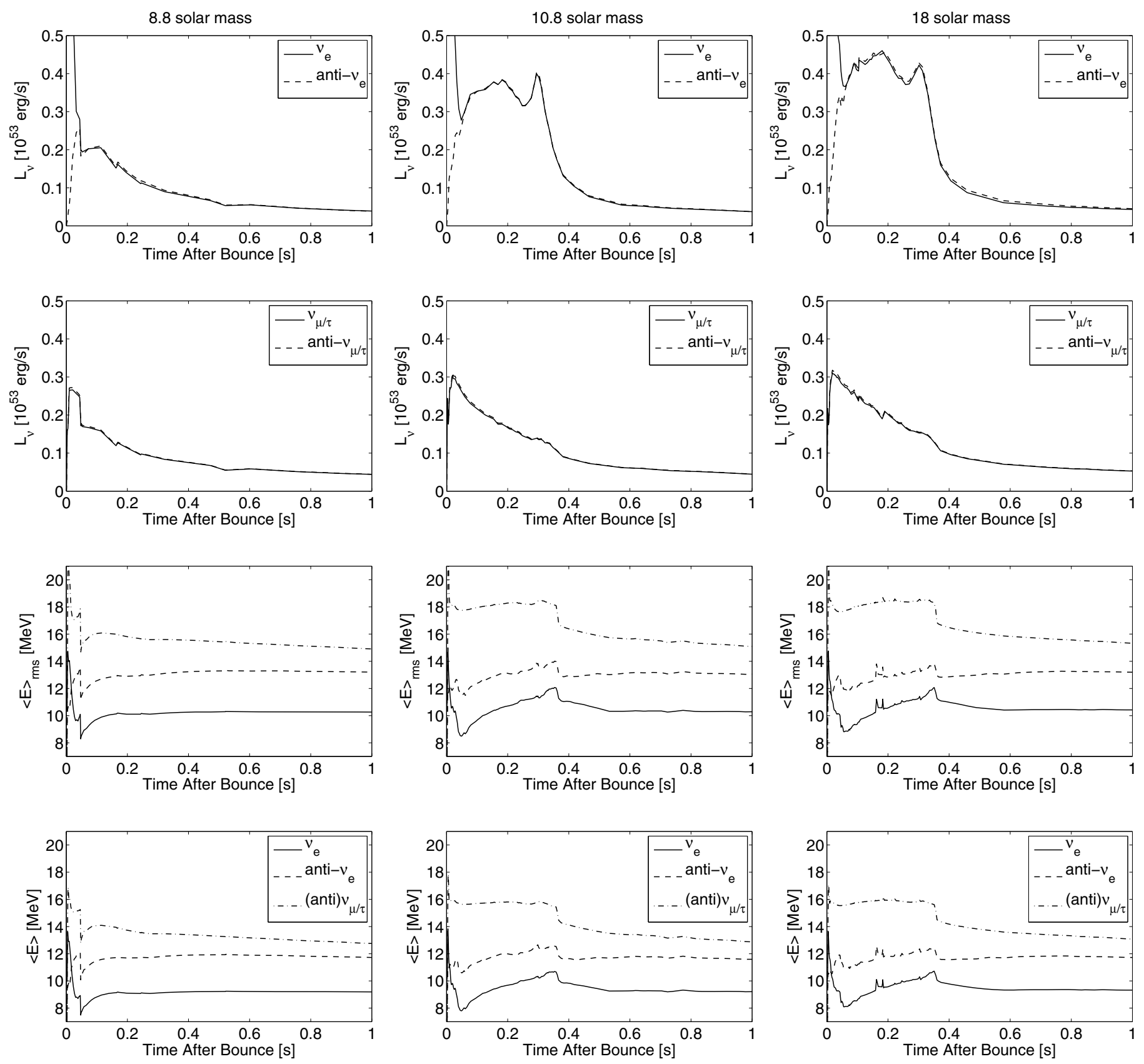

Fig. 2. Neutrino luminosities and energies with respect to time after bounce for the $8.8 \mathrm{M}_{\odot} \mathrm{O}-\mathrm{Ne}-\mathrm{Mg}$-core progenitor model from Nomoto (1983, 1984, 1987) (left panel) and the $10.8 \mathrm{M}_{\odot}$ and $18 \mathrm{M}_{\odot}$ Fe-core progenitor models from Woosley et al. (2002) (middle and right panels respectively), measured in a co-moving frame at $500 \mathrm{~km}$ distance.

about $0.15 \mathrm{M}_{\odot}$ of Fe-group nuclei are produced, where nuclear statistical equilibrium (NSE) applies (see Fig. 3a top panel). Instead, the central composition is dominated by ${ }^{16} \mathrm{O},{ }^{20} \mathrm{Ne}$ and ${ }^{24} \mathrm{Mg}$ nuclei. Because temperature and density increase during the collapse, these nuclei are burned into Fe-group nuclei and the NSE regime increases (see Fig. 3 middle panel). The core continues to deleptonize, which can be identified at the decreasing $Y_{\mathrm{e}}$ in Fig. 3. We use our nuclear reaction network as described in Sect. 2.2 to calculate the dynamically changing composition, based on the abundances provided by the progenitor model. The size of the bouncing core of $M_{\text {core }} \simeq 0.65 \mathrm{M}_{\odot}$ is significantly larger in comparison with the previous studies by Kitaura et al. (2006) and Liebendörfer (2004), illustrated in
Fig. 4 (left panel) at different velocity profiles before and at bounce. This is because we do not take the improved electron capture rates from Hix et al. (2003) and Langanke et al. (2003) into account, which are based on the capture of electrons at the distribution of heavy nuclei. It results in a lower central electron fraction at bounce and a consequently more compact bouncing core of about $\simeq 0.1 \mathrm{M}_{\odot}$, in comparison to the standard rates given in Bruenn (1985). The remaining difference is most likely due to the large asymmetry energy of the EoS from Shen et al. (1998) applied to the present study.

This progenitor is not only a special case due to the incomplete nuclear burning at the end of stellar evolution but also due to the steep density gradient which separates the dense core from 

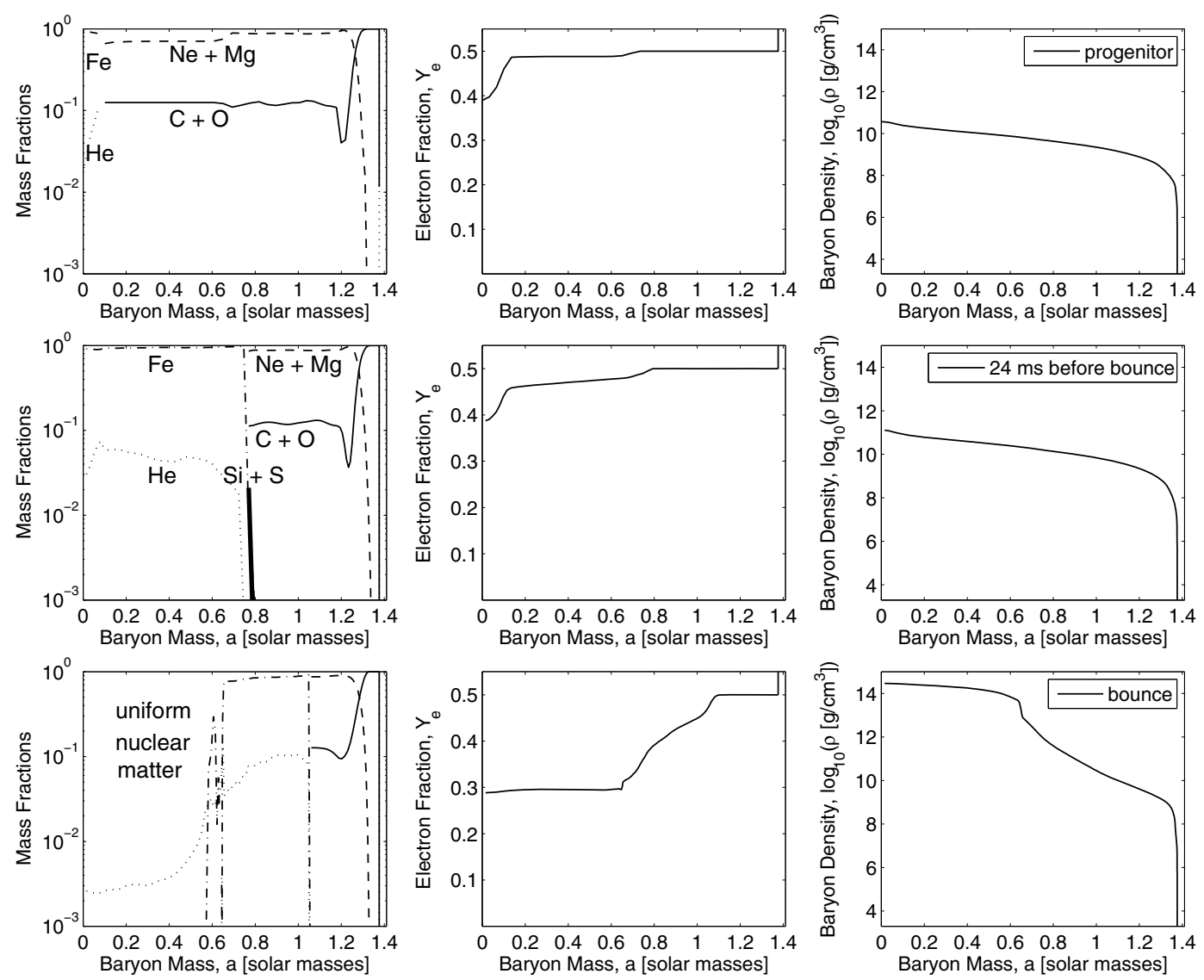

Fig. 3. Evolution of the $8.8 \mathrm{M}_{\odot}$ progenitor model from Nomoto $(1983,1984,1987)$ during the core collapse phase (top: progenitor configuration, middle: $24 \mathrm{~ms}$ before bounce, bottom: at bounce). The composition (left panels) are as follows: $\mathrm{C}+\mathrm{O}$ (thin solid line), $\mathrm{Ne}+\mathrm{Mg}(\mathrm{dashed} \mathrm{line),} \mathrm{Fe}+\mathrm{Ni}$ (dash-dotted line), $\mathrm{He}$ (dotted line), $\mathrm{Si}+\mathrm{S}$ (thick solid line).

the He- and H-rich envelope at $1.376 \mathrm{M}_{\odot}$, see Fig. 3c. There, the density drops over 13 orders of magnitude which makes it difficult to handle numerically.

The low density of the mass outside the O-Ne-Mg-core makes it possible to obtain the explosion in spherical symmetry supported via neutrino heating. Neutrino cooling in the region of dissociated nuclear matter causes the expanding shock front to turn into the SAS with no significant matter outflow. $v_{\mathrm{e}}$-cooling dominates over $\bar{v}_{\mathrm{e}}$-heating by one order of magnitude. Only at the dissociation line of infalling heavy nuclei, the neutrino energy deposition drives the SAS slowly to larger radii, for illustration see the heating(cooling) rates and velocity profile in Fig. 5 (left panel) at $20 \mathrm{~ms}$ post-bounce. However, the cooling of $v_{\mathrm{e}}$ still contributes to a large amount at $25 \mathrm{~ms}$ post-bounce over the heating of $\bar{v}_{\mathrm{e}}$ and $v_{\mu / \tau}$ in Fig. 5 (middle panel) behind the SAS. Only directly at the shock a low net-heating rate remains. Hence the influence of the neutrinos to the explosion itself is of minor importance. More important is the region of C-O-burning which produces $\mathrm{Ne}$ and $\mathrm{Mg}$. The hydrodynamic feedback to this thermodynamic transition can be identified already during the collapse phase of the progenitor core at the velocity profiles in Fig. 4 (left panel) at about $1.35-1.374 \mathrm{M}_{\odot}$. As material is shock heated post-bounce, the transition layer where $\mathrm{Ne}$ and $\mathrm{Mg}$ nuclei are burned into NSE propagates together with the expanding shock wave outwards. In other words, the Ne-Mg-layer of the progenitor is converted directly into NSE. Furthermore, the transition (discontinuity) from C-O-burning is falling quickly towards the SAS. It was found to be at about $350 \mathrm{~km}$ at $20 \mathrm{~ms}$ post-bounce and at about $200 \mathrm{~km}$ at $25 \mathrm{~ms}$ post-bounce, illustrated at the velocity profiles (bottom) in Fig. 5 (left-right panels). At about $30 \mathrm{~ms}$ post-bounce, the entire $\mathrm{Ne}-\mathrm{Mg}$-layer is converted into NSE due to the temperature increase obtained via shock heating. Hence, $\mathrm{C}$ and $\mathrm{O}$ nuclei are burned directly into NSE.

In contrast to more massive $\mathrm{Fe}$-core progenitors where O-burning produced an extended Si-S-layer, the amount of ${ }^{28} \mathrm{Si}$ and ${ }^{32} \mathrm{~S}$ is less than $1 \%$ at the end of nuclear burning for the $\mathrm{O}-\mathrm{Ne}-\mathrm{Mg}$-core discussed here (see Fig. 3a middle panel). This low fraction of $\mathrm{Si}$ and $\mathrm{S}$ is already converted into NSE during the initial collapse phase, due to the rapid density and temperature increase of the contracting core. Hence, $\mathrm{C}$ - and O-nuclei are burned directly into NSE during the post-bounce evolution. This sharp transition is related to a jump in the density and the thermodynamic variables. As the SAS propagates over this transition along the decreasing density, the shock accelerates to positive velocities (see Fig. 5 right panel). The consequent explosion is hence driven due to the shock propagation over the infalling transition between two different thermonuclear regimes rather than by pure neutrino heating, illustrated at the velocity profiles in Fig. 4 (right panel). Although Kitaura et al. (2006) approximated nuclear reactions during the evolution of the $\mathrm{O}-\mathrm{Ne}-\mathrm{Mg}$ core progenitor, the results of their explosion dynamics are in qualitative agreement with our findings.

The more massive Fe-core progenitors show the same thermo- and hydrodynamic features as discussed above for the $\mathrm{O}-\mathrm{Ne}-\mathrm{Mg}$-core due to the transition between different thermonuclear regimes. However, the differences are smaller because 
T. Fischer et al.: protoneutron star evolution and the neutrino-driven wind
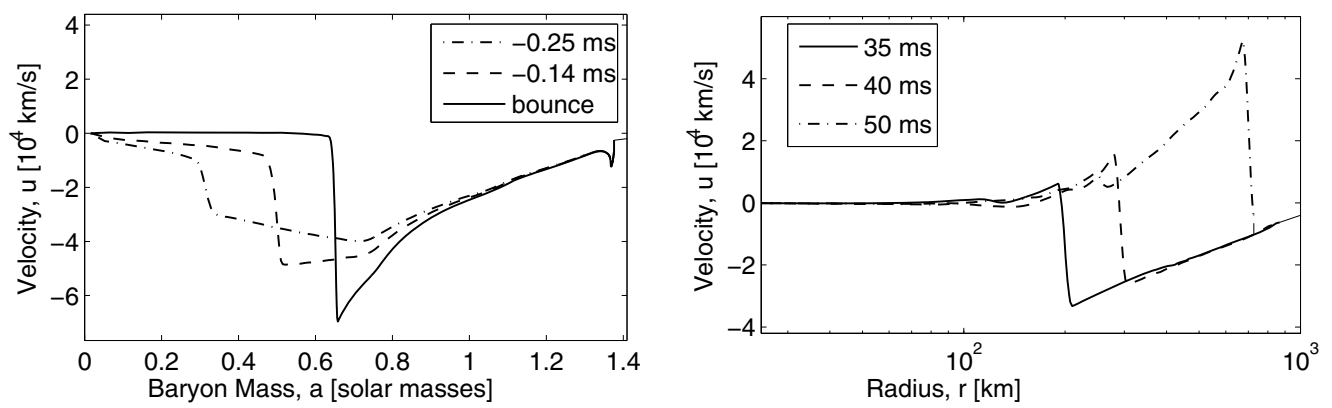

Fig. 4. Radial velocity profiles at selected times with respect to the baryon mass (left panel) before and at bounce and with respect to the radius (right panel) after bounce, for the $8.8 \mathrm{M}_{\odot}$ progenitor model from Nomoto $(1983,1984,1987)$.
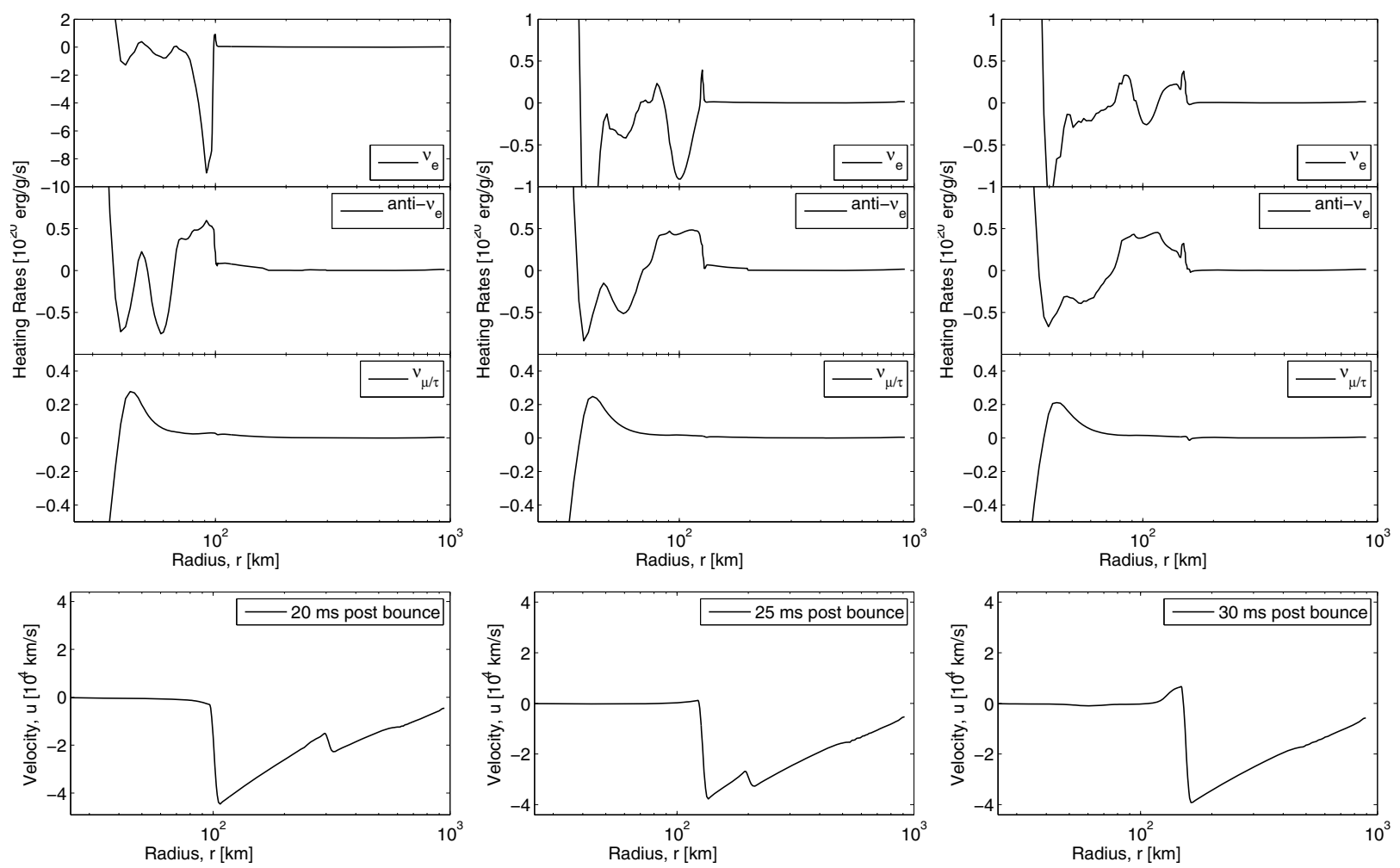

Fig. 5. Heating ( $>0)$ and cooling $(<0)$ rates for the $8.8 \mathrm{M}_{\odot}$ progenitor model from Nomoto $(1983,1984,1987)$ during the explosion phase at $20 \mathrm{~ms}$ (left panel), $25 \mathrm{~ms}$ (middle panel) and $30 \mathrm{~ms}$ (right panel) after bounce. For a better comparison, the velocities are plotted for the same post-bounce times.

C-O-burning produces an extended layer composed of ${ }^{28} \mathrm{Si}$ and ${ }^{32} \mathrm{~S}$. The transition of Si-burning into NSE is much smoother than the transition of C-O-burning into NSE. Furthermore, due to the more massive $\mathrm{Si}-\mathrm{S}$ and $\mathrm{C}-\mathrm{O}$-layers for the Fe-core progenitors, the transitions take more time on the order of seconds to fall onto the SAS. The presence of neutrino heating becomes important for the more massive $\mathrm{Fe}$-core progenitors to drive the SAS to large radii on a longer timescale. The effects of the shock propagation across the transition between different thermonuclear regimes has been pointed out in Bruenn et al. (2006) with respect to the explosion dynamics in axially-symmetric simulations of massive Fe-core progenitors. In our spherically symmetric models, we cannot confirm the driving force of explosions of $\mathrm{Fe}$-core progenitors to be the shock propagation across different thermonuclear regimes. We find that the explosions are initiated due to the deposition, although enhanced, of neutrino energy. The shock is accelerated additionally when crossing different thermonuclear regimes due to the density jumps at the transitions.

\subsection{Comparison of the neutrino spectra}

Striking is the agreement in the mean neutrino energies between all different progenitor models (including the $\mathrm{O}-\mathrm{Ne}-\mathrm{Mg}$-core and the Fe-core progenitors) during the explosion phase, although the neutrino emissivities and opacities are enhanced for the Fe-core progenitor models (see Fig. 2). The explosion phase for the O-Ne-Mg-core lasts only until about $40 \mathrm{~ms}$ post-bounce, which is significantly shorter in comparison to the more massive Fe-core progenitors. Furthermore, the luminosities are also lower by a factor of 2 . For all models, the electron antineutrino luminosity is higher than the electron neutrino luminosity on a timescale of $200 \mathrm{~ms}$ after the explosions have been launched. This slight difference reduces again at later times where the electron neutrino luminosity becomes again higher than the electron antineutrino luminosity. However, after the explosions have been launched the behaviors of the luminosities are in qualitative agreement for all models. The same holds for the mean neutrino energies which increase continuously during the neutrino 
(a)

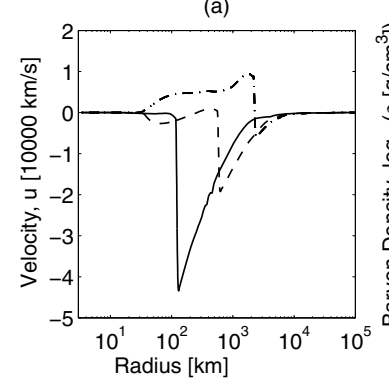

(c)

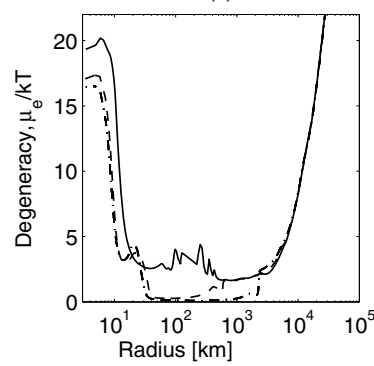

(b)

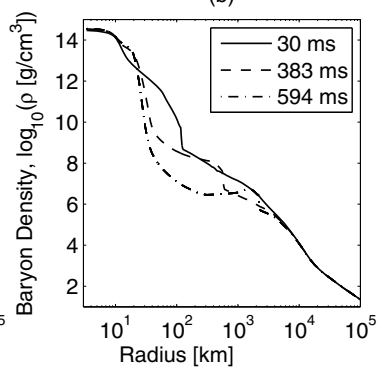

(d)

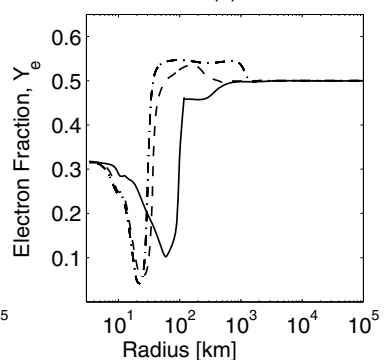

Fig. 6. Selected hydrodynamic variables during the initial explosion phase at three different post-bounce times for the $10.8 \mathrm{M}_{\odot}$ progenitor model from Woosley et al. (2002).

heating phase. The electron (anti)neutrinos have rms energies of about 12 (14) MeV where as after the explosions have been launched, rms energies of about 11 (13) MeV are obtained. The values remain constant on the timescale of $1 \mathrm{~s}$ post-bounce. The $(\mu / \tau)$-neutrinos have rms energies of about $18 \mathrm{MeV}$ during the neutrino heating phase and about $15 \mathrm{MeV}$ after the explosion has been launched. These differences in the mean neutrino energies and luminosities during the neutrino heating, initial and proceeding explosion phases are in correspondence with the electron fraction of the material, as will be illustrated in the following section.

\subsection{The electron fraction of the early ejecta}

During the neutrino heating phase, the neutrino spectra are mainly determined by mass accretion at the neutrinospheres. Neutron-rich nuclei from the progenitor star with an electron fraction of $Y_{\mathrm{e}} \simeq 0.45$ are falling onto the oscillating SAS and dissociate into free nucleons and light nuclei, see Fig. 6d. These nucleons accrete then slowly onto the PNS surface at the center. Due to the increased electron-degeneracy behind the SAS in Fig. 6c, weak-equilibrium is established at a lower value of the electron fraction of $Y_{\mathrm{e}} \leq 0.15$.

As soon as the SAS is revived and propagates outward, see the velocity and density profiles in Figs. $6 a$ and $b$, the electron degeneracy behind the expanding shock is reduced and weakequilibrium is established at a higher value of the electron fraction of $Y_{\mathrm{e}}>0.56^{3}$. The capture rates for electron-neutrinos at neutrons are favored over electron-antineutrino captures at protons. This slight difference results in an electron and hence proton excess. Consequently the explosion ejecta are found to be initially proton-rich. This behavior of the electron fraction was

3 The EoS from Shen et al. (1998) is limited to a maximum electron fraction of $Y_{\mathrm{e}} \geq 0.564$. The EoS has been extended by Gögelein (2007, priv. comm.) to model asymmetric nuclear matter with an electron fraction above 0.564 . We assume an ideal gas of free nucleons and light nuclei, which is a sufficient assumption for the conditions found in the region of the extremely proton-rich ejecta. found for all our explosion models, for the 10.8 and $18 \mathrm{M}_{\odot}$ Fe-core progenitors with artificially enhanced opacities and for the O-Ne-Mg-core using the standard opacities. Such explosion models were investigated with respect to the nucleosynthesis in general and with respect to the $v p$-process by Fröhlich et al. (2006a-c).

One of the main goals of the present investigation is to determine the behavior of the electron fraction for the initially protonrich ejecta on a long timescale on the order of $10 \mathrm{~s}$, in a consistent manner. We explore the question if the material ejected in the neutrino-driven wind becomes neutron-rich and which are the conditions (e.g. entropy per baryon, expansion timescale) obtained in the neutrino-driven wind. These aspects are of special relevance for the composition of the ejecta, which is determined via explosive nucleosynthesis analysis, in particular in order to be able to draw conclusions with respect to a possible site for the production of heavy elements via the $r$-process. Therefore, the continued expansion of the explosion ejecta must be simulated, for which the inclusion of a large physical domain of the progenitor up to the He-layer is required. Furthermore, since the electronic charged current reaction rates and the neutrino fluxes determine the electron fraction, the PNS contraction at the center and hence the contraction of the neutrinospheres are essential.

\section{The neutrino-driven wind}

In this section we investigate the post explosion evolution with special focus on the properties of the ejecta, in particular the electron fraction. We explore the problem if the initially protonrich ejecta become neutron-rich at later times on the order of $10 \mathrm{~s}$ and if the conditions might indicate a possible site for the nucleosynthesis of heavy nuclei via the $r$-process. This has been assumed in static steady-state as well as parametrized dynamic wind models, based on the results obtained by Woosley et al. (1994). The ejected material in their simulations does never become proton-rich, the electron fraction was found to continuously decrease with time after the explosion has been launched. Thus, the ejecta were investigated in a region where the conditions are favorable for the $r$-process. The stellar models applied to the present investigation of the neutrino driven wind are the $8.8 \mathrm{M}_{\odot} \mathrm{O}-\mathrm{Ne}-\mathrm{Mg}$-core and the 10.8 and $18 \mathrm{M}_{\odot} \mathrm{Fe}$-core progenitors, where for the latter two cases the explosions are obtained using the artificially enhanced opacities as described in Sect. 2.3.

After the explosions have been launched, the region between the expanding explosion shock and the neutrinospheres cools rapidly and the density decreases continuously as illustrated in Figs. 7 and $8 \mathrm{~d}$ and $\mathrm{h}$. In order to determine the evolution of the electron fraction $Y_{\mathrm{e}}$, the non-local neutrino fluxes are as important as the local neutrino reaction rates. Since the PNS and hence the neutrinospheres contract continuously due to the deleptonization, the degeneracy increases and matter at the PNS surface is found to be extremely neutron-rich with $Y_{\mathrm{e}} \leq 0.1$ (see Figs. 7 and $8 \mathrm{~d}$ and f).

Independent of the progenitor model, the region on top of the PNS surface is continuously subject to neutrino heating during the post explosion phase. The dominant heating sources are the absorption of electron-(anti)neutrinos at free nucleons, due to the high fraction of free nucleons (dissociated nuclear matter) present in the region on top of the PNS, as shown in Fig. 9. The neutrino pair production and thermalization processes have a negligible contribution to the heating outside the neutrinospheres. In order to compare the heating and cooling rates in Fig. 9, we plot the quantities with respect to the baryon density. While neutrino cooling is still dominantly present at 
(a)

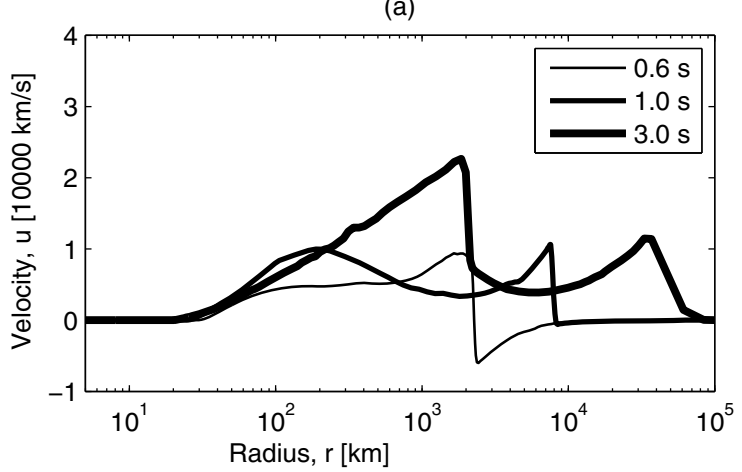

(c)

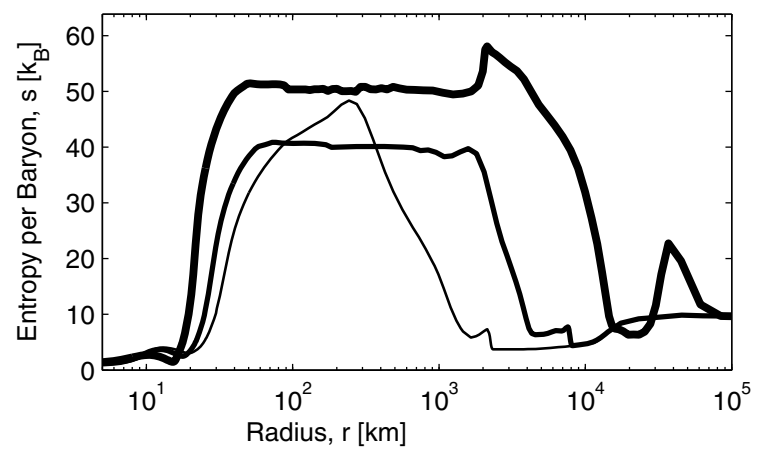

(e)

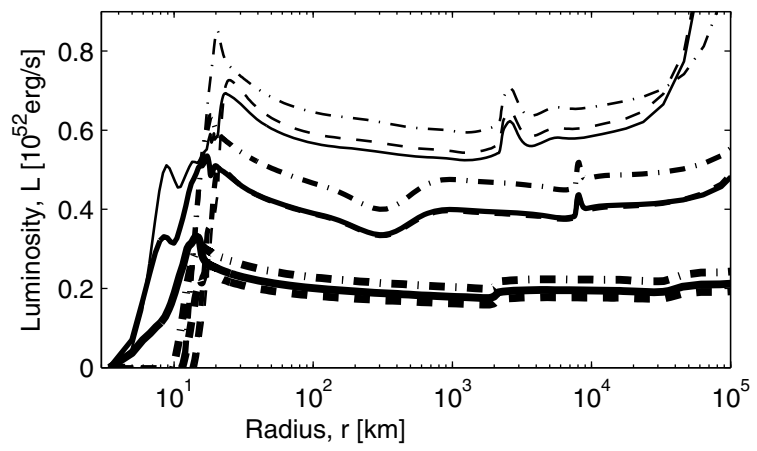

(g)

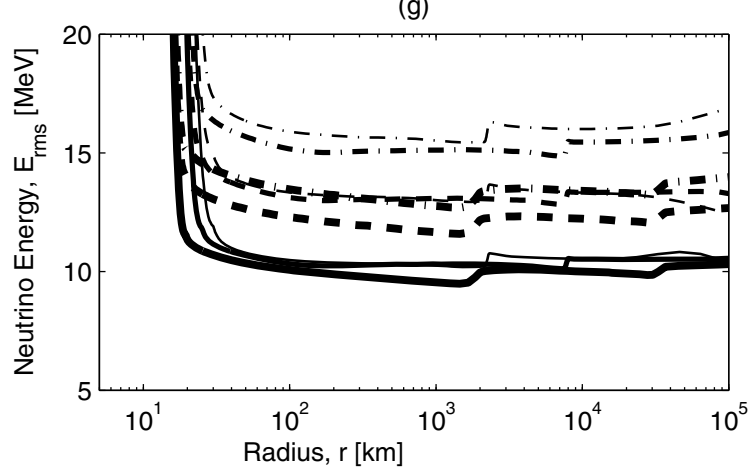

(b)

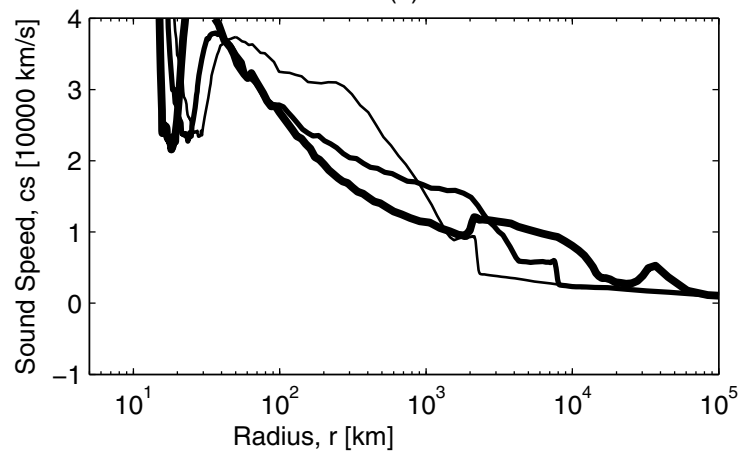

(d)

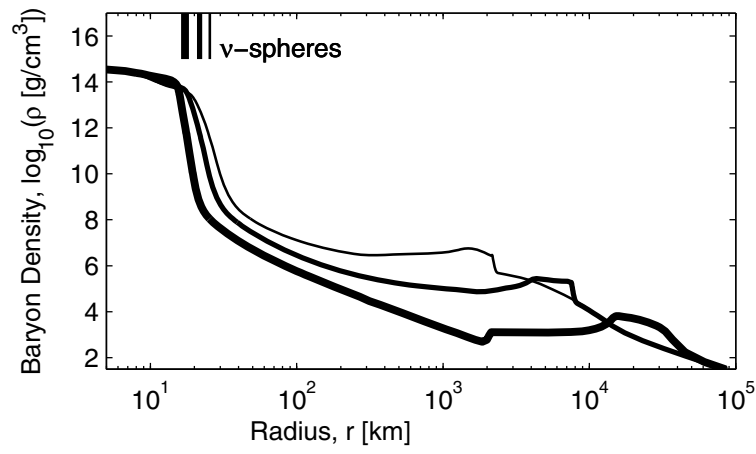

(f)

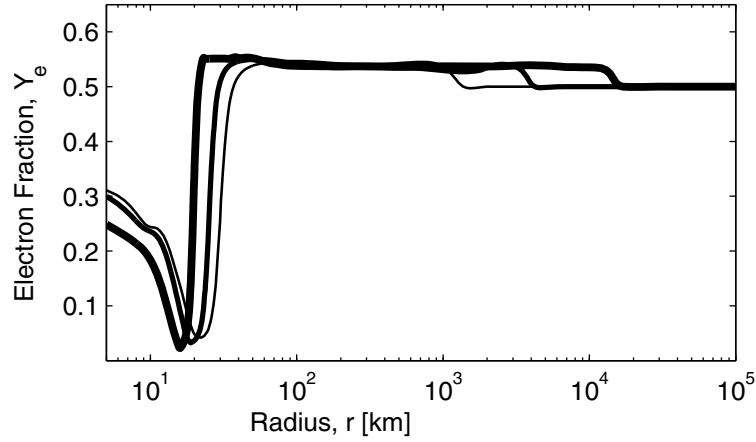

(h)

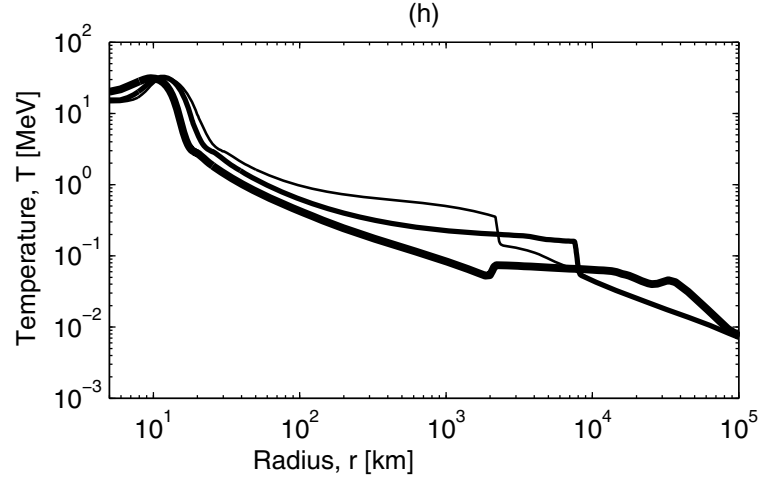

Fig. 7. Selected hydrodynamic variables during the formation of the neutrino-driven wind at three different post-bounce times for the $10.8 \mathrm{M}_{\odot}$ progenitor model from Woosley et al. (2002). In addition, graphs e) and g) show the neutrino luminosities and rms neutrino energies (solid lines: $v_{\mathrm{e}}$, dashed lines: $\bar{v}_{\mathrm{e}}$, dash-dotted lines: $v_{\mu / \tau}$ ). For this progenitor model the neutrino-driven wind becomes supersonic, using the enhanced opacities.

$\sim 500 \mathrm{~ms}$ post-bounce (thin lines in Fig. 9), at later times after $\sim 1$ s post-bounce (thick lines in Fig. 9) neutrino cooling vanishes and only heating is found in the density domain of interest, i.e. between $10^{7}-10^{12} \mathrm{~g} / \mathrm{cm}^{3}$. Figures $7 \mathrm{~d}$ and $8 \mathrm{~d}$ show the conditions for the contracting PNSs at the center via the radial baryon density profiles and the electron-neutrinospheres. The region of interest where the neutrino-driven wind develops corresponds to the density domain of $10^{7}-10^{11} \mathrm{~g} / \mathrm{cm}^{3}$. The degeneracy of the early ejecta favors proton-rich matter where a high electron fraction of $Y_{\mathrm{e}} \simeq 0.54$ is obtained. Hence, the absorption of electronantineutrinos at free protons dominates over electron-neutrino absorption at free neutrons. The corresponding radial neutrino 
(a)

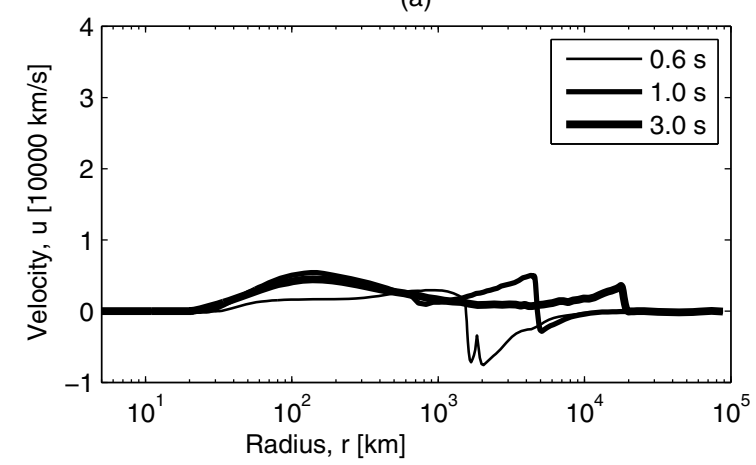

(c)

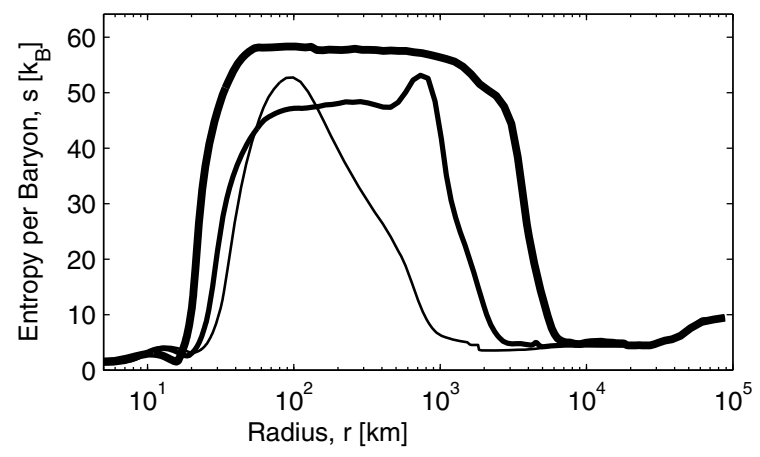

(e)

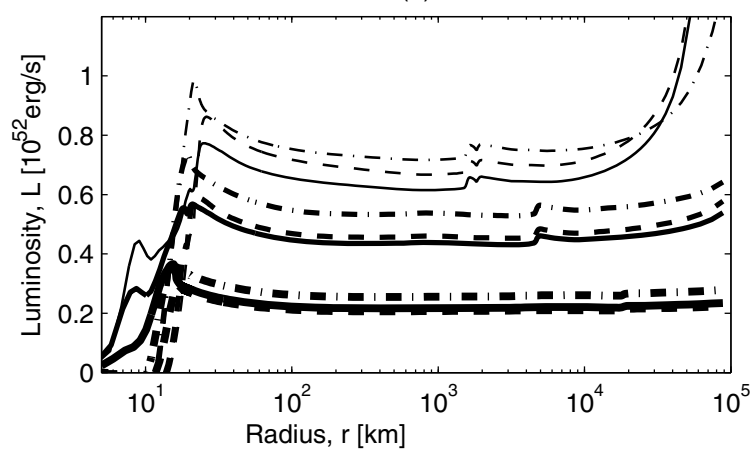

(g)

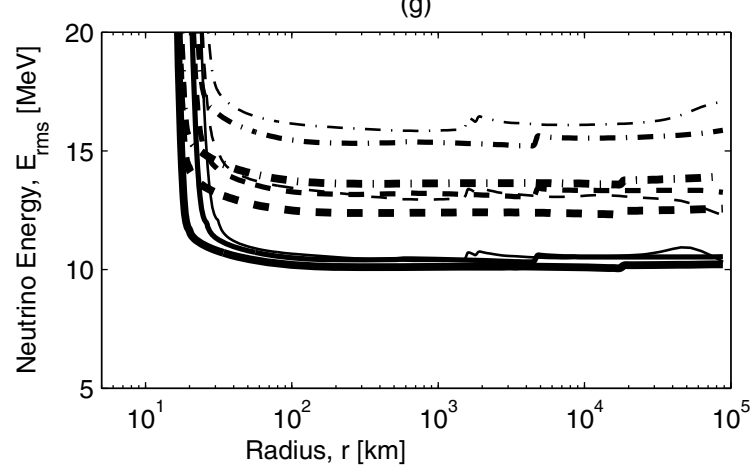

(b)

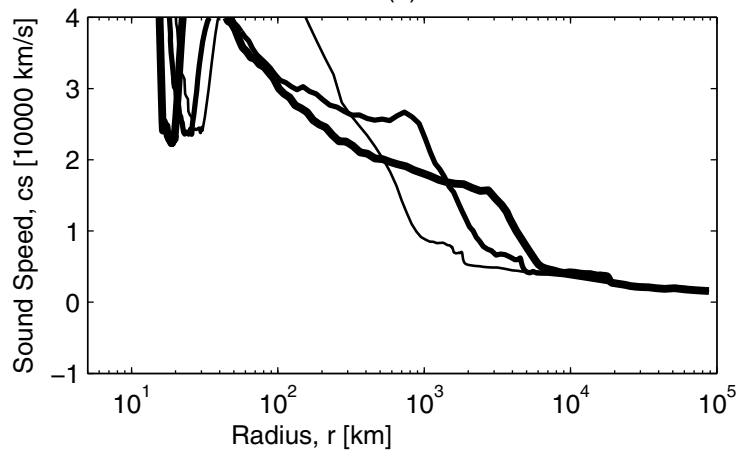

(d)

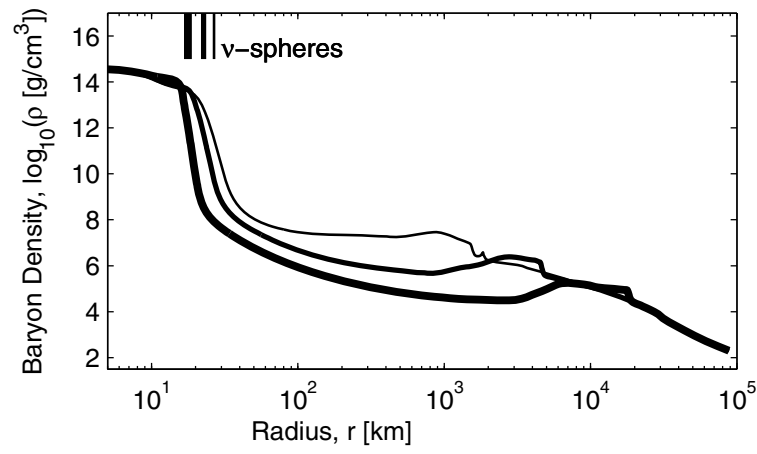

(f)

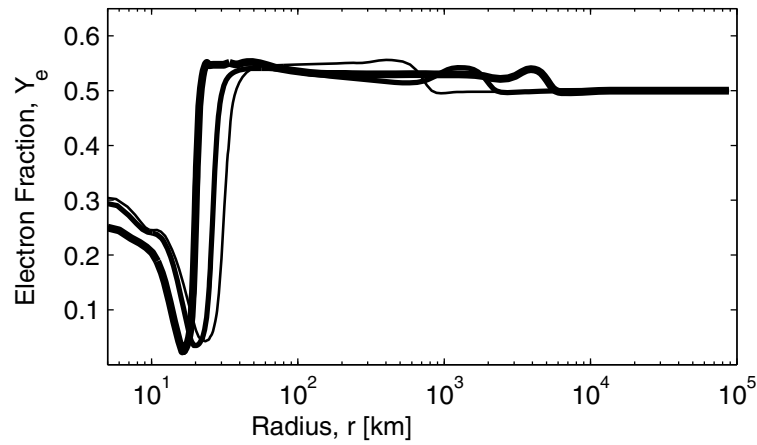

(h)

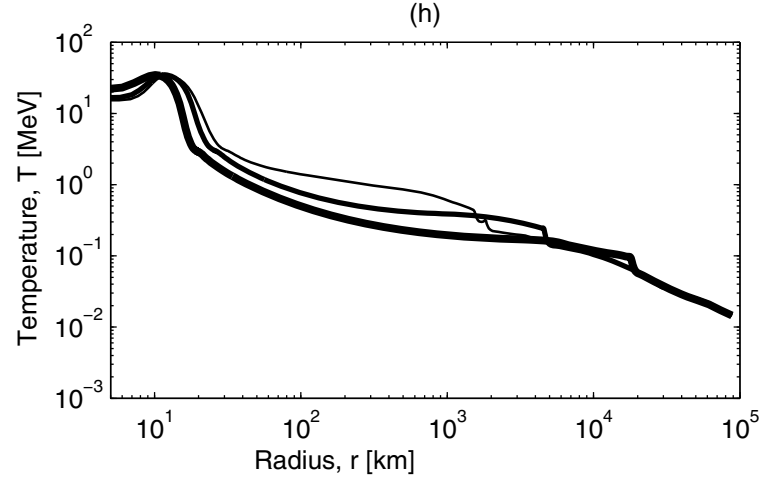

Fig. 8. The same configuration as Fig. 7 for the $18 \mathrm{M}_{\odot}$ progenitor model from Woosley et al. (2002). The neutrino-driven wind remains subsonic for this progenitor model, even using the enhanced opacities.

luminosities and rms energies are shown in Figs. 7 and 8e and g. In addition, for the first time we were able to follow the deleptonization burst from core bounce for several seconds over a large physical domain including several $10^{5} \mathrm{~km}$ of the progenitor star. The outrunning luminosity peak can be identified in the luminosities in Figs. 7 and 8 e at $0.6 \mathrm{~s}$ after bounce at a distance between $5 \times 10^{4}$ and $10^{5} \mathrm{~km}$, leaving the computational domain between $1-2 \mathrm{~s}$ post-bounce.
After the explosions have been launched, the continued energy transfer from the neutrino radiation field into the fluid outside the neutrinospheres as illustrated in Fig. 9 drives the matter entropies to high values, shown in Figs. 7 and 8c. The heat deposition at the PNS surface accelerates matter to positive velocities, see Figs. 7 and $8 \mathrm{a}$, after $\simeq 1$ s post-bounce. This matter outflow is known as the neutrino-driven wind, which proceeds adiabatically at larger radii. This is consistent with the 
(a) $v_{e}$

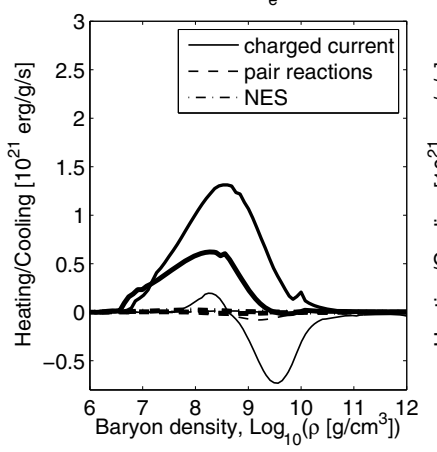

(b) anti-v

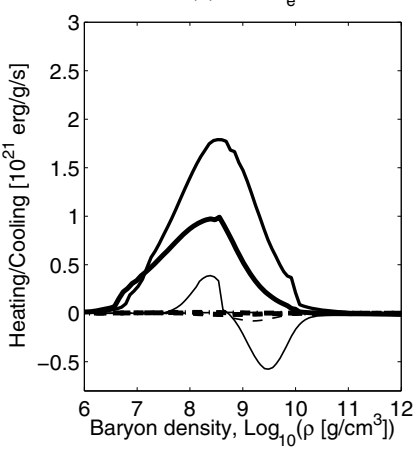

Fig. 9. Heating and cooling rates with respect to the baryon density at three different post-bounce times $0.5 \mathrm{~s}$ (thin lines), $1 \mathrm{~s}$ (intermediate lines), $5 \mathrm{~s}$ (thick lines) for the $10.8 \mathrm{M}_{\odot}$ progenitor model from Woosley et al. (2002).

constant radial entropy per baryon profiles in the graphs (c). Furthermore, the rapidly decreasing luminosities reach values below $5 \times 10^{51} \mathrm{erg} / \mathrm{s}$ already $1 \mathrm{~s}$ after bounce (see Figs. 7 and $8 \mathrm{e}$ ). The luminosities continue to decrease and reach values below $1 \times 10^{51} \mathrm{erg} / \mathrm{s}$ at $10 \mathrm{~s}$ after bounce. The mean neutrino energies also decrease constantly where values below $10 \mathrm{MeV}$ for the electron-flavor neutrinos and below $12 \mathrm{MeV}$ for the $(\mu / \tau)$-neutrinos are obtained (see Figs. 7 and $8 \mathrm{~g}$ ).

Several previous wind studies achieved supersonic matter outflow velocities for the neutrino-driven wind due to assumed high luminosities. In any case, the accelerated material of the neutrino-driven wind collides with the slower and subsonically expanding explosion ejecta. Comparing Figs. 7 and 8, the more compact wind region of the $18 \mathrm{M}_{\odot}$ progenitor model produces a less pronounced neutrino-driven wind in comparison to the $10.8 \mathrm{M}_{\odot}$ progenitor model. The densities of the wind region are higher up to two orders of magnitude and the temperatures are higher by a factor of 2 . The resulting velocities of the neutrinodriven wind outflow are lower by a factor of 2 and stay below $10^{4} \mathrm{~km} \mathrm{~s}^{-1}$. Hence, the neutrino-driven wind remains subsonic for all times for the $18 \mathrm{M}_{\odot}$ progenitor model (see Fig. 8a) where the wind develops supersonic velocities for the $10.8 \mathrm{M}_{\odot}$ progenitor model (see Fig. 7a). In the case of a supersonic neutrinodriven wind, this leads to the formation of the reverse shock known as the wind termination shock. The formation of the wind termination shock of the $10.8 \mathrm{M}_{\odot}$ progenitor model is illustrated in Fig. 10 and will be discussed in the following paragraph. In order to analyze the dynamical evolution and the consequences of the formation of the reverse shock, steady-state wind models cannot be used. Radiation hydrodynamics is required in order to describe the dynamical effects consistently. Our results, obtained using general relativistic radiation hydrodynamics based on spectral three-flavor Boltzmann neutrino transport, are in qualitative agreement with the detailed parametrized investigation by Arcones et al. (2007).

During the initial and subsonic wind expansion, the matter entropies in Fig. 10c increase slowly from 4 to 5-10 $k_{\mathrm{B}} /$ baryon and the densities in Fig. 10b and temperatures in Fig. 10e decrease on a long timescale over several seconds. Furthermore, the reduced degeneracy in the wind increases the electron fraction shown in Fig. 10d slowly on the same timescale. When the material is accelerated supersonically with velocities of several $10^{4} \mathrm{~km} \mathrm{~s}^{-1}$ up to radii of a several $10^{3} \mathrm{~km}$ (see Figs. 10a and f), the entropies increase from $s \simeq 5-10 k_{\mathrm{B}} /$ baryon to $s \simeq 40-60 k_{\mathrm{B}} /$ baryon on a short timescale of the order of $100 \mathrm{~ms}$.

During this rapid expansion, the density and temperature decrease drastically from $10^{10} \mathrm{~g} / \mathrm{cm}^{3}$ to $10^{4}-10^{2} \mathrm{~g} / \mathrm{cm}^{3}$ and from $3 \mathrm{MeV}$ to $0.1-0.01 \mathrm{MeV}$ respectively (see Figs. $10 \mathrm{~b}$ and e). It also corresponds to a rapid decrease of the degeneracy which in turn is reflected in a rapid increase of the electron fraction of the accelerated material on top of the PNS surface, from $Y_{\mathrm{e}} \simeq 0.1$ to $Y_{\mathrm{e}} \simeq 0.56$ (see Fig. 10d). Furthermore, the supersonically expanding neutrino-driven wind collides with the explosion ejecta as can bee seen in Fig. 10a (solid red line) at radii of several $10^{4} \mathrm{~km}$. Consequently, the previously accelerated material decelerates behind the explosion ejecta as can be seen in the velocities in Fig. 10f. This phenomenon becomes significant after about $2 \mathrm{~s}$ post-bounce and corresponds to the formation of the reverse shock, i.e. the wind termination shock. (See Fig. 10a dashed red line at radii of several $10^{3} \mathrm{~km}$.) It causes an additional entropy increase to the final values of $s \simeq 50-100 k_{\mathrm{B}}$ /baryon. During the rapid deceleration on the same short timescale on the order of $100 \mathrm{~ms}$, the densities in Fig. 10b and temperatures in Fig. 10e increase again slightly, where the degeneracy increases and hence the electron fraction reduces slightly to values of $Y_{\mathrm{e}} \simeq$ 0.54 . The following dynamical evolution is given by the subsonic and adiabatic expansion of the explosion ejecta on a longer timescale on the order of seconds. The density and temperature decrease slowly where the entropies of $s \simeq 50-100 k_{\mathrm{B}} /$ baryon and the electron fraction of about $Y_{\mathrm{e}}=0.54$ remain constant. The latter aspects are essential for the nucleosynthesis analysis of the ejecta. It can be understood in the sense that the neutrino reaction rates freeze out and the matter conditions correspond to the neutrino free streaming regime.

Note that the strong neutrino-driven wind for the $10.8 \mathrm{M}_{\odot}$ progenitor model is obtained using the enhanced opacities as introduced in Sect. 2.3. We additionally illustrate selected properties of the neutrino-driven wind for the $8.8 \mathrm{M}_{\odot}$ progenitor model in Fig. 11 where a strong neutrino-driven wind was obtained using the standard emissivities and opacities given in Bruenn (1985). This is due to the low density of the region between the neutrinospheres at the PNS surface and the expanding explosion shock, where neutrino heating via the standard rates and energy from nuclear burning are sufficient to drive a strong supersonic matter outflow. Matter entropies increase to $s \simeq 10 k_{\mathrm{B}}$ /baryon during the initial acceleration of the wind and the densities and temperatures decrease slowly on a timescale of seconds. The properties during the initial acceleration observed are similar to those of the more massive $10.8 \mathrm{M}_{\odot} \mathrm{Fe}$-core progenitor. The same holds for the acceleration to supersonic velocities. The timescale is reduced to $100 \mathrm{~ms}$ where the entropies increase rapidly to $s \simeq 20-50 k_{\mathrm{B}}$ /baryon (see Fig. 11c) and due to the reduced degeneracy the electron fraction increases from $Y_{\mathrm{e}}=0.1$ at the PNS surface to $Y_{\mathrm{e}}=0.56$ (see Fig. 11d). Density and temperature decrease to $10-100 \mathrm{~g} / \mathrm{cm}^{3}$ and $0.001 \mathrm{MeV}$ respectively (see Figs. $11 \mathrm{~b}$ and e). The difference to the more massive $10.8 \mathrm{M}_{\odot} \mathrm{Fe}$-core progenitor is due to the lower mass enclosed between the PNS surface and the expanding explosion ejecta. For the more massive $10.8 \mathrm{M}_{\odot} \mathrm{Fe}$-core progenitor in Fig. 10f, the previously accelerated material collides with the explosion ejecta already after a few $100 \mathrm{~ms}$. Here the supersonic wind expands on a much longer timescale up to several seconds before it collides with the explosion ejecta (see Fig. 11f). During this adiabatic expansion, entropy and electron fraction remain constant. The fast material collides with the much slower expanding explosion ejecta so that the material is decelerated and the reverse shock appears. This is again similar to the formation of the reverse shock for the more massive $10.8 \mathrm{M}_{\odot}$ Fe-core progenitor as discussed above. Matter entropies increase 
(a)

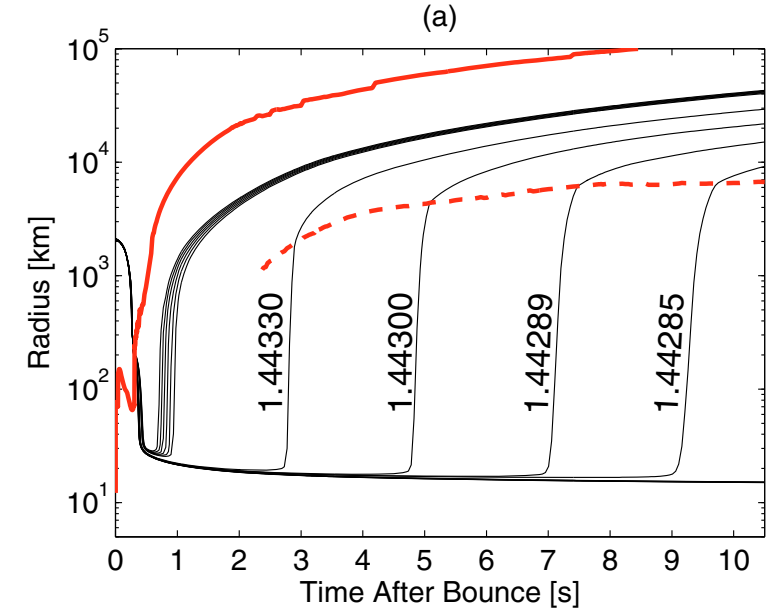

(c)

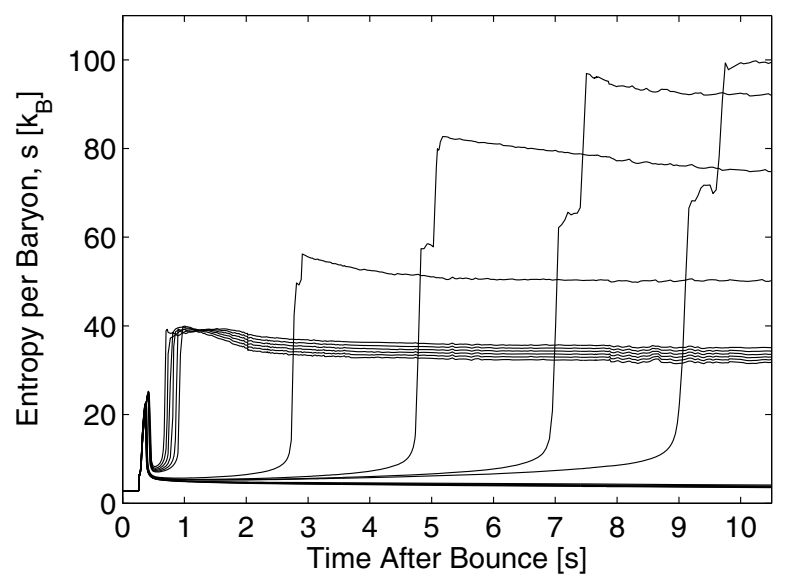

(e)

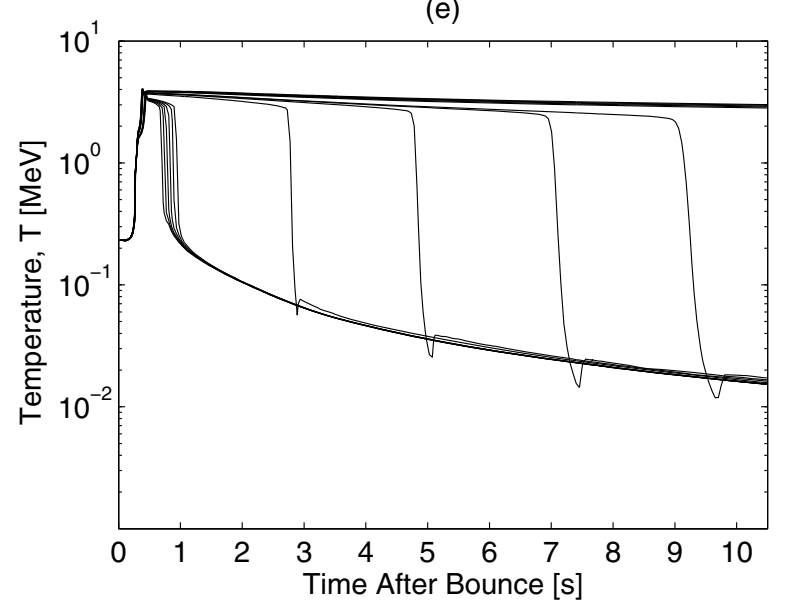

(b)

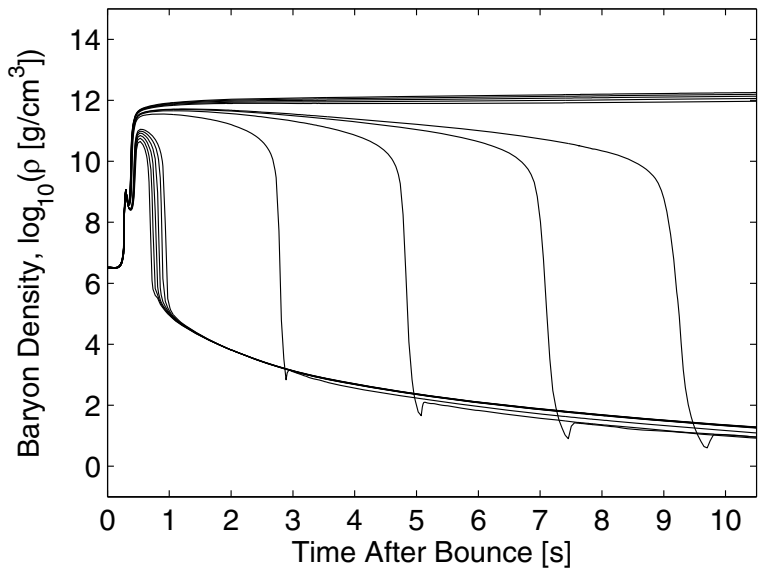

(d)

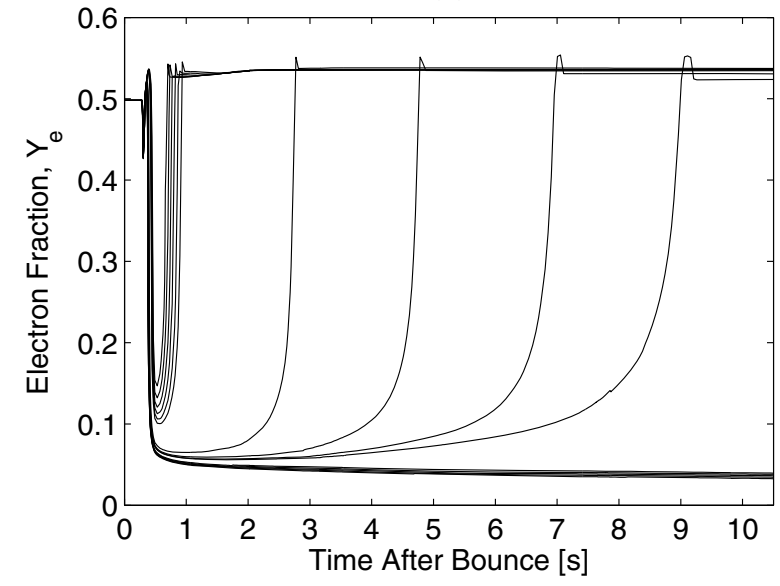

(f)

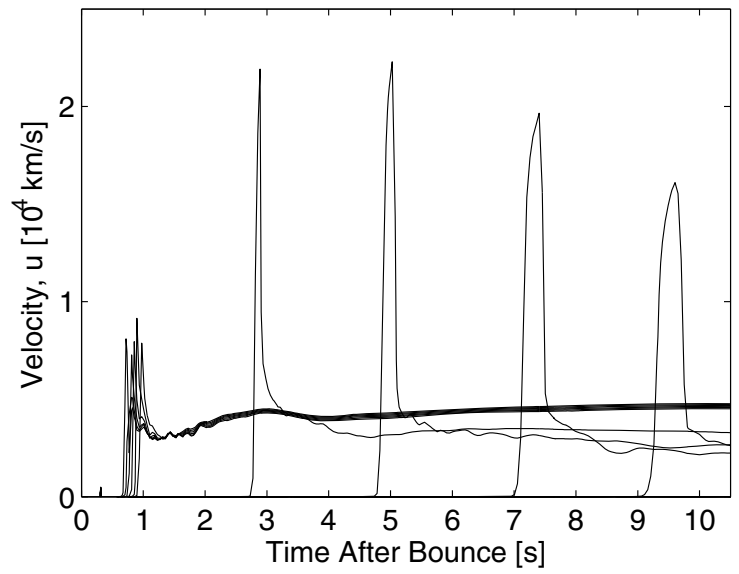

Fig. 10. Evolution of selected mass elements in the neutrino-driven wind (as listed in graph a) from 1.44285-1.44450 $\mathrm{M}_{\odot}$ baryon mass) for the $10.8 \mathrm{M}_{\odot}$ progenitor model from Woosley et al. (2002) where the enhanced opacities are used. Graph a) shows in addition the position of the expanding explosion shock (red solid line) and the position of the wind termination shock (red dashed line).

to $s=100 k_{\mathrm{B}}$ /baryon (see Fig. 11c), density and temperature increase sightly (see Figs. $11 \mathrm{~b}$ and e) and the electron fraction reduces slightly to $Y_{\mathrm{e}} \simeq 0.52-0.54$ due to the increased degeneracy (see Fig. 11d). The following evolution is determined by the adiabatic expansion of the explosion ejecta during which the entropy and electron fraction remain constant.
In the following paragraph, we will discuss the composition of the neutrino-driven wind region to some extent. This is possible due to the recently implemented nuclear reaction network. It includes the free nucleons and the symmetric nuclei from ${ }^{4} \mathrm{He}$ to ${ }^{56} \mathrm{Ni}$ plus ${ }^{53} \mathrm{Fe},{ }^{54} \mathrm{Fe}$ and ${ }^{56} \mathrm{Fe}$. The initial composition is given by the progenitor model. Mostly ${ }^{28} \mathrm{Si}$ and ${ }^{30} \mathrm{~S}$ are 
(a)

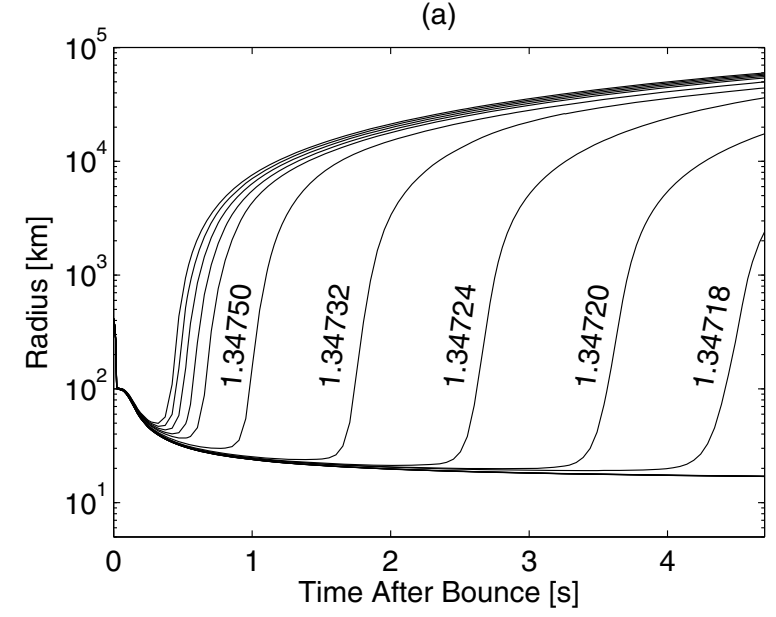

(c)

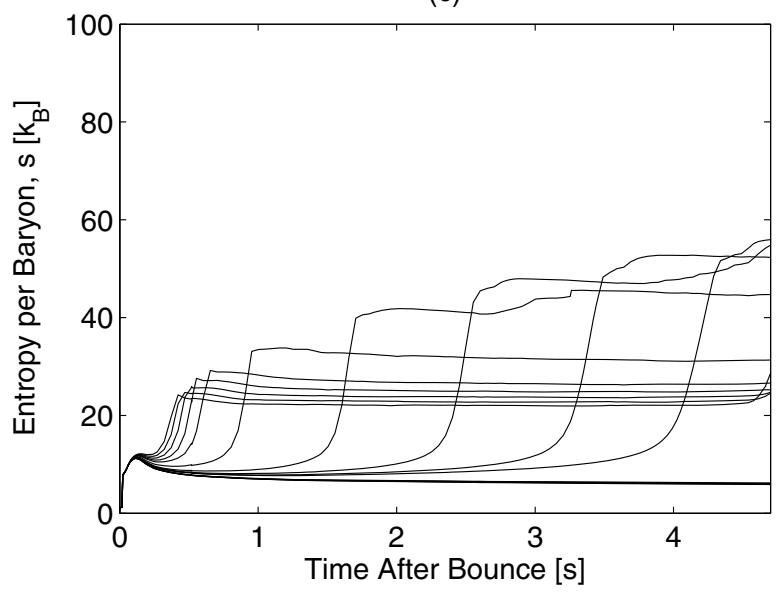

(e)

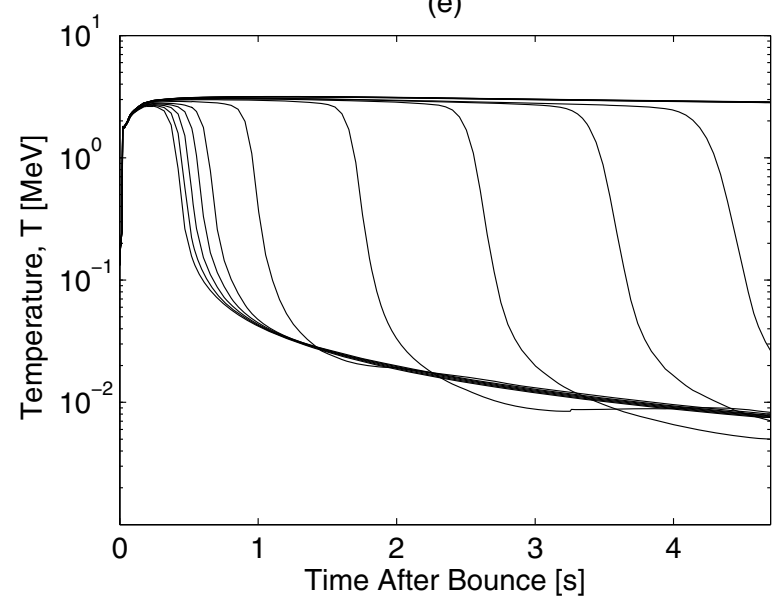

(b)

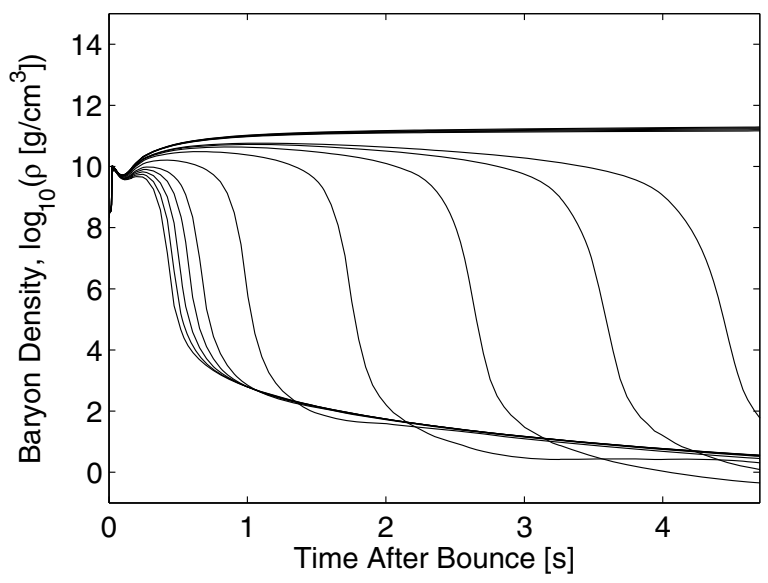

(d)

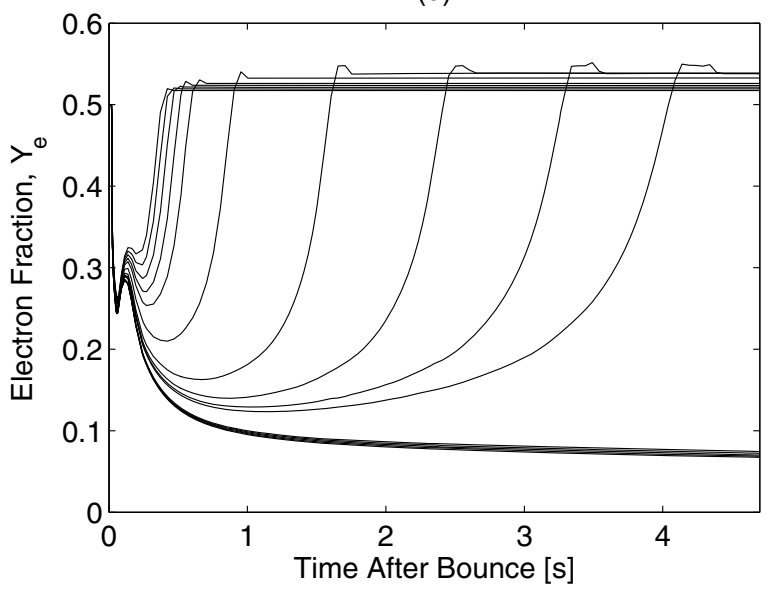

(f)

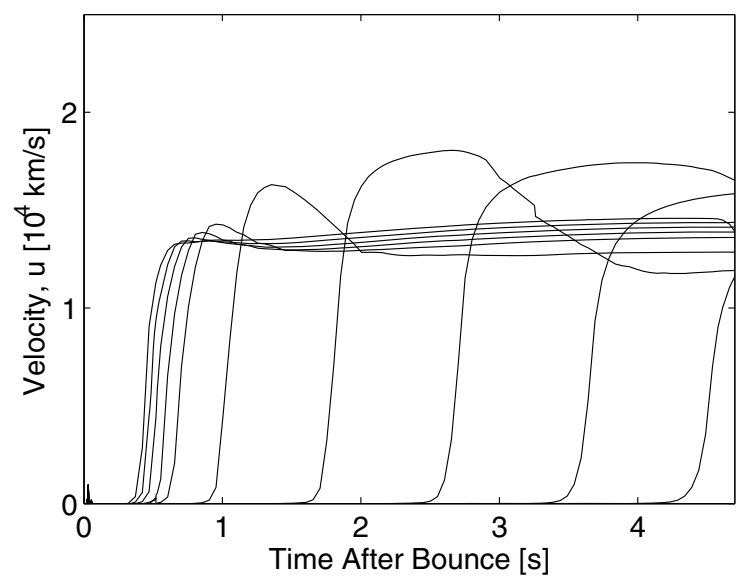

Fig. 11. Evolution of selected mass shells in the neutrino-driven wind (as listed in graph a) from 1.34718-1.34750 $\mathrm{M}_{\odot}$ baryon mass) for the $8.8 \mathrm{M}_{\odot}$ progenitor model from Nomoto $(1983,1984,1987)$ where the standard emissivities and opacities given in Bruenn (1985) are used. The graphs show the same configurations as Fig. 10.

shock-heated and burned to Fe-group nuclei due to the temperature and density jump during the initial expansion of the explosion shock (see Fig. 12 and compare with Figs. 7 and 8d and $f$ ). The high fraction of these Fe-group nuclei reduces behind the explosion shock due to photodisintegration, indicated by the region of low density and high entropy in Figs. 7 and 8b and c. This produces a high fraction of $\alpha$-particles, which in our model represent light nuclei. The region of $\alpha$-particle domination behind the expanding explosion shock increases with time. This behavior is illustrated in Fig. 12 for both Fe-core progenitor 

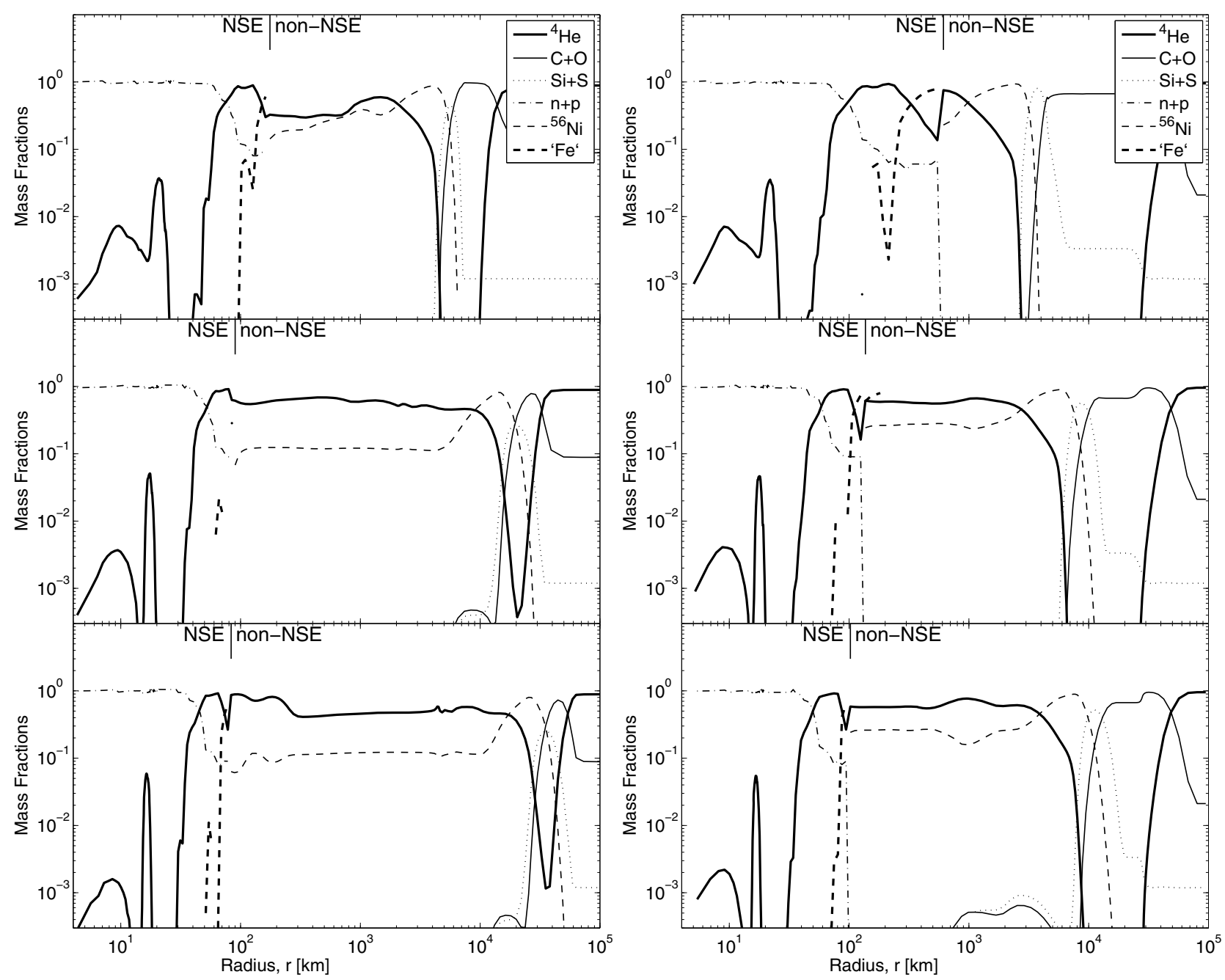

Fig. 12. Selected radial mass fraction profiles during the neutrino driven wind phase for the $10.8 \mathrm{M}_{\odot}$ (left panel) and the $18 \mathrm{M}_{\odot}$ (right panel) progenitor model from Woosley et al. (2002) at $1 \mathrm{~s}$ (top), $3 \mathrm{~s}$ (middle) and $5 \mathrm{~s}$ (bottom) post-bounce. The vertical lines represent the separation of NSE (EoS for hot and dense nuclear matter) where heavy nuclei are represented by a single Fe-group nucleus "Fe" with average atomic mass and charge and non-NSE (nuclear reaction network) where the most abundant Fe-group element is ${ }^{56} \mathrm{Ni}$, at temperatures of $\simeq 0.5 \mathrm{MeV}$.

models under investigation. The position of the explosion shock coincides with the maximum of the mass fraction of Fe-group nuclei (in particular ${ }^{56} \mathrm{Ni}$ ). In addition, density and temperature of the neutrino-driven wind on top of the PNS surface decrease continuously with time. The low temperatures and densities in that region do not justify the assumption of NSE beyond $\sim 1 \mathrm{~s}$ after bounce, where temperatures reach values below $0.5 \mathrm{MeV}$. Instead, our nuclear reaction network is used to determine the composition in that region. The decreasing density and temperature and the presence of a high fraction of free nucleons favor the freeze out of light nuclei. Finally, the entire region between the expanding explosion shock and the PNS surface is found to be dominated in our simulations by $\alpha$-particles. In Fig. 12, the radii of the NSE to non-NSE transitions are indicated by vertical lines. The slight mismatch between the abundances between the heavy "Fe"-group nuclei (the representative heavy nucleus with average atomic mass and charge in NSE) and ${ }^{56} \mathrm{Ni}$ (nonNSE) as well as between the $\alpha$ 's is due to the different nuclear models used for the two regimes. While in NSE the EoS for hot and dense nuclear matter assumes ${ }^{56} \mathrm{Fe}$ as the most stable nucleus due to the lowest mass per nucleon for low temperatures and densities, the nuclear reaction network applied in non-NSE calculates the composition dynamically based on tabulated reaction rates.

\section{Comparison with previous wind studies}

\subsection{The proton-to-baryon ratio of the wind}

The most fundamental approximations made in previous wind studies is the simplified description of the radiationhydrodynamics equations, see for example Duncan et al. (1986) and Qian \& Woosley (1996). More crucial is the absence of neutrino transport. Neutrino heating and cooling is calculated based on parametrized neutrino luminosities and mean energies. Hence, such models explore the neutrino-driven wind by varying the neutrino luminosities and energies, where the simplified radiation-hydrodynamics equations are solved (see for example Thompson et al. 2001). Since neutrino transport is neglected, the evolution equation for the electron fraction Eq. (15) cannot be solved consistently because the neutrino distribution functions are unknown. In the following paragraph, we will discuss the assumptions made for the evolution of the electron fraction in the neutrino-driven wind which go back to Qian \& Woosley (1996). 
T. Fischer et al.: protoneutron star evolution and the neutrino-driven wind

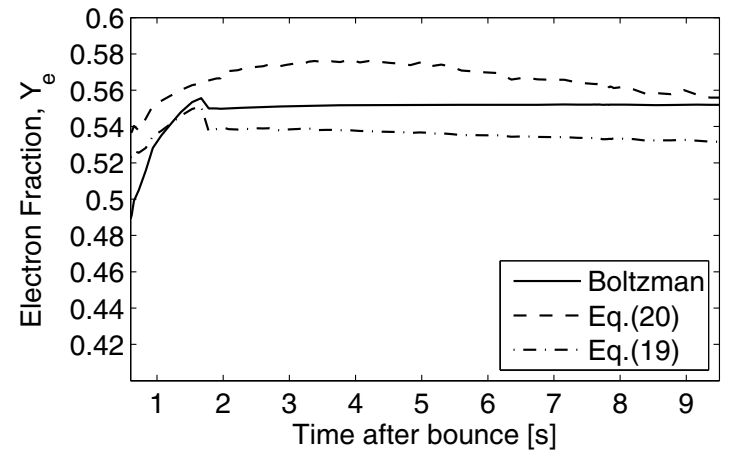

(a) Different electron fraction approximations (dashdotted line: Eq. (19), dashed line: Eq. (20)) in comparison with Boltzmann transport (solid line).

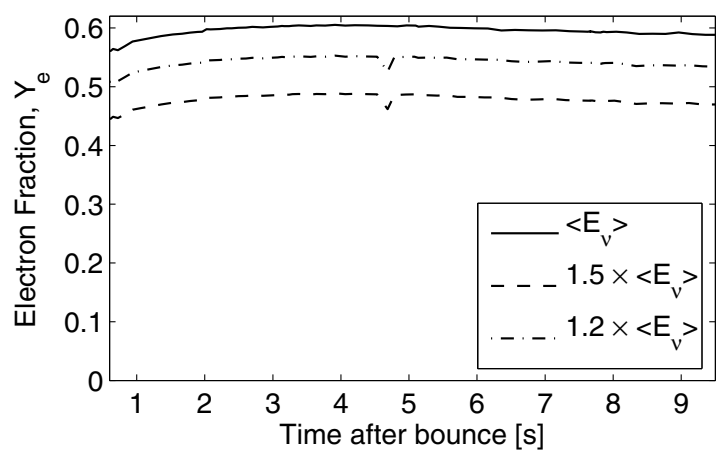

(b) Evolution of the electron fraction according to Eq. (20). The three lines correspond to different assumptions for the electron antineutrino mean energy.

Fig. 13. The Electron fraction approximations at a distance of $10 \mathrm{~km}$ outside the electron-neutrinosphere for the $10.8 \mathrm{M}_{\odot}$ progenitor model from Woosley et al. (2002).

Applying the theory of weak interactions based on the reaction rates $\lambda_{i j}$ for the reaction partners $(i, j)$, i.e. electron and positron as well as electron neutrino and antineutrino captures, the evolution equations for the electron and positron fractions can be written as follows

$\frac{\mathrm{d} Y_{\mathrm{e}^{-}}}{\mathrm{d} t}=-\lambda_{\mathrm{e}^{-} \mathrm{p}} Y_{\mathrm{e}^{-}} Y_{\mathrm{p}}+\lambda_{v_{\mathrm{e}} \mathrm{n}} Y_{v_{\mathrm{e}}} Y_{\mathrm{n}}$

$\frac{\mathrm{d} Y_{\mathrm{e}^{+}}}{\mathrm{d} t}=-\lambda_{\mathrm{e}^{+} \mathrm{n}} Y_{\mathrm{e}^{+}} Y_{\mathrm{n}}+\lambda_{\bar{v}_{\mathrm{e}}} Y_{\bar{v}_{\mathrm{e}}} Y_{\mathrm{p}}$

These expressions can be combined to calculate the evolution of the total number of charges, using the relations $Y_{\mathrm{p}}=Y_{\mathrm{e}}$ and $Y_{\mathrm{n}}=1-Y_{\mathrm{e}}$,

$$
\begin{aligned}
\frac{\mathrm{d} Y_{\mathrm{e}}}{\mathrm{d} t}= & \frac{\mathrm{d}}{\mathrm{d} t}\left(Y_{\mathrm{e}^{-}}-Y_{\mathrm{e}^{+}}\right)=\lambda_{\mathrm{e}^{+} \mathrm{n}} Y_{\mathrm{e}^{+}}+\lambda_{v_{\mathrm{e}} \mathrm{n}} Y_{v_{\mathrm{e}}} \\
& -\left(\lambda_{\mathrm{e}^{-} \mathrm{p}} Y_{\mathrm{e}^{+}}+\lambda_{\mathrm{e}^{+} \mathrm{n}} Y_{\mathrm{e}^{+}}+\lambda_{v_{\mathrm{e}} \mathrm{n}} Y_{v_{\mathrm{e}}}+\lambda_{\overline{\mathrm{e}}_{\mathrm{e}}} Y_{\bar{v}_{\mathrm{e}}}\right) Y_{\mathrm{e}},
\end{aligned}
$$

assuming fully dissociated nuclear matter. This expression is approximated in a crucial but powerful way (Qian \& Woosley 1996, Eq. (73)), ignoring contributions from electron and positron captures as well as the decoupling of radiation from matter and the angular dependency of the neutrino distribution function on the distance from the energy-dependent neutrinospheres, as follows

$$
\begin{aligned}
Y_{\mathrm{e}} & \simeq \frac{\lambda_{\mathrm{e}^{+} \mathrm{n}} Y_{\mathrm{e}^{+}}+\lambda_{v_{\mathrm{e}} \mathrm{n}} Y_{v_{\mathrm{e}}}}{\lambda_{\mathrm{e}^{-} \mathrm{p}} Y_{\mathrm{e}^{+}}+\lambda_{\mathrm{e}^{+} \mathrm{n}} Y_{\mathrm{e}^{+}}+\lambda_{v_{\mathrm{e}} \mathrm{n}} Y_{v_{\mathrm{e}}}+\lambda_{\overline{\mathrm{e}}_{\mathrm{e}}} Y_{\bar{v}_{\mathrm{e}}}} \\
& \simeq \frac{\lambda_{v_{\mathrm{e}} \mathrm{n}} Y_{v_{\mathrm{e}}}}{\lambda_{v_{\mathrm{e}} \mathrm{n}} Y_{v_{\mathrm{e}}}+\lambda_{\bar{v}_{\mathrm{e}} \mathrm{p}} Y_{\bar{v}_{\mathrm{e}}}} .
\end{aligned}
$$

This approximation of the electron fraction was further simplified and expressed in terms of the neutrino luminosities $L_{v}$ and $\left\langle\epsilon_{v}\right\rangle$, which is the ratio of mean-square energy to average energy, and the well known rest mass difference between neutron and proton $Q=m_{\mathrm{n}}-m_{\mathrm{p}}=1.2935 \mathrm{MeV}$, as follows

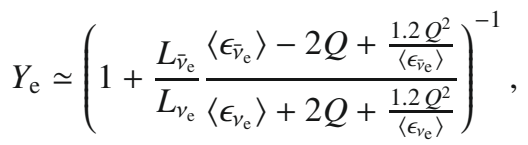

which is used in previous static steady-state and parametrized dynamic studies of the neutrino-driven wind.
Figure 13a compares the electron fraction behavior at a distance of $10 \mathrm{~km}$ outside the electron-neutrinosphere, from Boltzmann neutrino transport (solid line) with the approximations Eq. (19) based on the neutrino capture rates (dashed line) and Eq. (20) based on the luminosities and mean neutrino energies (dash-dotted lines). The approximations are in qualitative agreement with Boltzmann transport. The differences on the longer timescale are most likely due to the presence of light and heavy nuclei which are not taken into account explicitly in the approximations. They change the number of free nucleons available for the reactions in Eqs. (16) and (17). All descriptions agree qualitatively in the prediction of a generally proton-rich material in the wind, based on the neutrino spectra obtained via Boltzmann transport.

\subsection{The neutrino observables in the wind}

Comparing the neutrino spectra in Fig. 14 with the spectra assumed in previous static steady-state and dynamic wind studies (see for example Thompson et al. 2001; and Arcones et al. 2007), we find two major differences: One, the neutrino luminosities and mean neutrino energies assumed are significantly higher than those we find and two, the assumed behavior with respect to time is different.

The commonly used assumptions made in static steady-state and parametrized dynamic wind studies go back to the detailed investigation from Woosley et al. (1994), who performed core collapse simulations based on sophisticated input physics. They investigated the neutrino-driven explosion of a $20 \mathrm{M}_{\odot}$ progenitor star and followed the evolution for $18 \mathrm{~s}$ post-bounce into the neutrino-driven wind phase. In their simulations the electron (anti)neutrino luminosities decreased from initially $4 \times 10^{52}$ $\left(3 \times 10^{52}\right) \mathrm{erg} / \mathrm{s}$ at the onset of the explosion to $6 \times 10^{51}$ $\left(7.5 \times 10^{50}\right) \mathrm{erg} / \mathrm{s}$ at $10 \mathrm{~s}$ after bounce, where strictly $L_{\bar{\gamma}_{\mathrm{e}}}>L_{v_{\mathrm{e}}}$ after the onset of the explosion. The difference between the neutrino and antineutrino luminosities remained small and constant with respect to time up to $3 \mathrm{~s}$ post-bounce and increased only significantly after $4-5$ s post-bounce, after which the difference reached its maximum of $1.5 \times 10^{50} \mathrm{erg} / \mathrm{s}$ at the end of the simulation at about $18 \mathrm{~s}$ post-bounce. The electron flavor neutrino luminosities in our models follow a different behavior. They reach $1 \times 10^{51} \mathrm{erg} / \mathrm{s}$ at about 5,6 and $8 \mathrm{~s}$ post-bounce for the $8.8,10.8$ and $18 \mathrm{M}_{\odot}$ progenitor models respectively. The higher electron flavor neutrino luminosities for the more massive progenitors are 

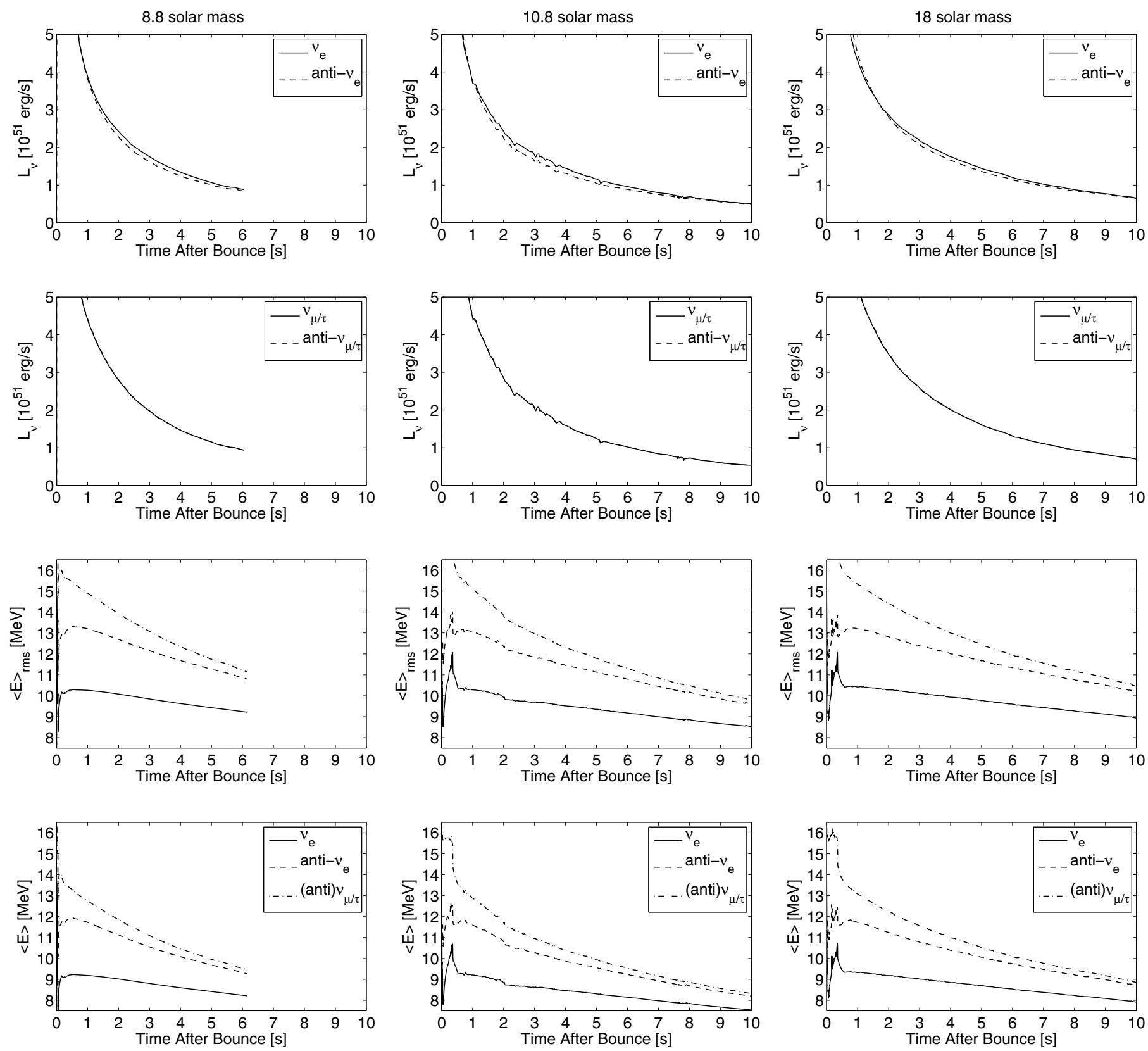

Fig. 14. Neutrino luminosities and mean energies with respect to time after bounce for the $8.8 \mathrm{M}_{\odot} \mathrm{O}-\mathrm{Ne}-\mathrm{Mg}$-core from Nomoto (1983, 1984, 1987) (left panel) and the $10.8 \mathrm{M}_{\odot}$ (middle panels) and $18 \mathrm{M}_{\odot}$ (right panel) Fe-core progenitor models from Woosley et al. (2002), measured in the co-moving reference frame at a distance of $500 \mathrm{~km}$.

in correlation with the more massive PNSs and the hence larger number of neutrinos emitted. However, the difference between electron-neutrino and electron-antineutrino luminosities found in the present investigation is significantly lower than the difference in Woosley et al. (1994). During the initial explosion phase until about $300 \mathrm{~ms}$ after the onset of the explosion, the electron antineutrino luminosity is slightly higher than the electron neutrino luminosity by about $1 \times 10^{50} \mathrm{erg} / \mathrm{s}$ which in our models explains the electron fraction of $Y_{\mathrm{e}}>0.5$ of the early explosion ejecta. After about $900 \mathrm{~ms}$ post-bounce, the luminosities can hardly be distinguished where during the initial neutrino-driven wind phase after about $1 \mathrm{~s}$ after bounce the electron neutrino luminosity becomes higher than the electron antineutrino luminosity by about $1 \times 10^{50} \mathrm{erg} / \mathrm{s}$. This difference reduces again at later times at about $6 \mathrm{~s}$ post-bounce and the electron flavor neutrino luminosities become more and more similar (see Fig. 14).
Even more different are the values and the behavior of the mean neutrino energies, see Fig. 14 and compare with Fig. 2 of Woosley et al. (1994). They found $(\mu / \tau)$-neutrino energies of about $35 \mathrm{MeV}$ which remained constant with respect to time. Their electron-antineutrino energies increased slightly from about $20 \mathrm{MeV}$ to $22 \mathrm{MeV}$ where the electron-neutrino energies decrease from $14 \mathrm{MeV}$ to $12 \mathrm{MeV}$. This increasing difference between the electron neutrino and antineutrino spectra favored neutron-rich material, which was consistent with their findings of $Y_{\mathrm{e}}<0.5$ for the material ejected in the neutrinodriven wind in Woosley et al. (1994). We cannot confirm these results for the mean neutrino energies nor the evolution of the spectra. In contrast, all mean neutrino energies decrease with respect to time for all our models. This is a consequence of lepton number and energy loss of the central PNS where the neutrinos diffuse out. The electron (anti)neutrino energies 
decrease from about $10(12) \mathrm{MeV}$ at the onset of the explosion to about $8.5(9) \mathrm{MeV}$ and the $(\mu / \tau)$-neutrino energies decrease from $16 \mathrm{MeV}$ to $10 \mathrm{MeV}$ at the end of the simulations. Hence, not only the mean energies decrease also the difference between the electron flavor neutrino spectra decreases. The reason for the neutrino spectra to become more similar with respect to time is related to the evolution of the thermodynamic properties at the neutrinospheres, and will be discussed in the following subsection.

\subsection{The PNS contraction}

The behavior of the neutrino spectra and hence the evolution and the properties of the neutrinospheres is related to the PNS contraction. The contraction is caused by a continuous deleptonization and translates to a continued steepening of the density gradient at the PNS surface. Hence, the neutrinosphere radii for the electron flavor neutrinos move closer together with time. The evolution of the neutrinosphere radii for both electron neutrino and antineutrino are illustrated in Fig. $15 \mathrm{a}$ for the $10.8 \mathrm{M}_{\odot}$ progenitor model. Their difference reduces from $740 \mathrm{~m}$ at about $1 \mathrm{~s}$ post-bounce to $370 \mathrm{~m}$ at about $5 \mathrm{~s}$ post-bounce and further to $260 \mathrm{~m}$ at about $10 \mathrm{~s}$ post-bounce.

This contraction behavior has consequences for the neutrino spectra, which are determined during the neutrino-driven wind phase by diffusion rather than by mass accretion. Hence, the electron flavor neutrino luminosities can be determined as follows

$L_{v}=\left.\frac{1}{4} 4 \pi r^{2} u_{v}\right|_{R_{v}}$

where $u_{v} \propto T^{4}$ is the thermal black body spectrum for ultrarelativistic fermions with temperature $T$. The matter temperatures at the neutrinospheres decrease with respect to time as shown in Fig. 15b. This is due to the continued loss of lepton number and energy, carried away by the diffusing neutrino radiation field as illustrated in Fig. 16 for the $10.8 \mathrm{M}_{\odot}$ from Woosley et al. (2002). The lepton number decreases from $Y_{L} \simeq 0.3$ at $2 \mathrm{~s}$ post bounce to $Y_{L} \simeq 0.18$ at $10 \mathrm{~s}$ post-bounce, see Fig. 16c. The additionally reduced mean neutrino energies (on average), from $\langle E\rangle_{\mathrm{rms}} \simeq 150 \mathrm{MeV}$ to $\langle E\rangle>_{\mathrm{rms}} \simeq 50 \mathrm{MeV}$ (see Fig. 16d), and the consequent reduced temperature-peak inside the PNS (see Fig. 16b) cause the contraction of the outer layers of the PNS. This can be identified via the density increase in Fig. 16a. Note in addition to the maximum temperature decrease at the outer layers of the PNS, the central temperature increases from $18 \mathrm{MeV}$ to $23 \mathrm{MeV}$ on the post-bounce times between 2 and $10 \mathrm{~s}$. This is caused by the contraction of the deleptonizing outer layers of the PNS which compresses the central part. The evolution of the reducing temperature at the neutrinospheres is shown in Fig. 15b. In combination with the loss of leptons number, it explains the decreasing electron flavor neutrino luminosities and mean neutrino energies with respect to time. Furthermore, the temperature difference decreases with respect to time from $0.467 \mathrm{MeV}$ at about $1 \mathrm{~s}$ post-bounce to $0.362 \mathrm{MeV}$ at about $10 \mathrm{~s}$ post-bounce. Consequently the neutrino spectra become more similar with respect to time. As illustrated in Fig. 14, the difference in the electron flavor neutrino luminosities and mean neutrino energies decreases for all models under investigation. In addition, Figs. $15 \mathrm{c}$ and $\mathrm{d}$ illustrate the evolution of the baryon density and the electron fraction at the corresponding neutrinospheres. It becomes additionally clear from the electron fraction approximation Eq. (20), that it is not the absolute values for the mean neutrino and antineutrino energies that determine whether matter becomes neutron- or protonrich but their ratio.

Since this difference is small in our simulations, with initially $\left\langle E_{v_{\mathrm{e}}}\right\rangle_{\mathrm{rms}} \simeq 10 \mathrm{MeV}$ and $\left\langle E_{\bar{\nu}_{\mathrm{e}}}\right\rangle_{\mathrm{rms}} \simeq 13 \mathrm{MeV}$ (at about $1 \mathrm{~s}$ postbounce) and only $\left\langle E_{v_{\mathrm{e}}}\right\rangle_{\mathrm{rms}} \simeq 9 \mathrm{MeV}$ and $\left\langle E_{\bar{\gamma}_{\mathrm{e}}}\right\rangle_{\mathrm{rms}} \simeq 11 \mathrm{MeV}$ (at later times at $10 \mathrm{~s}$ post-bounce), the values found for the electron fraction of $Y_{\mathrm{e}}>0.5$ (solid line in Fig. 13b for the $Y_{\mathrm{e}}$-approximation based on the luminosity and mean neutrino energies) clearly illustrate that the accelerated matter in the neutrino driven wind stays proton-rich for more than $10 \mathrm{~s}$. This is in qualitative agreement with Boltzmann transport as discussed above and shown in Fig. 13a. Hence we find Eq. (20) to be a good approximation to model the electron fraction in the wind. On the other hand, most of the previous studies select the neutrino luminosities and mean energies to investigate a neutronrich neutrino driven wind. In order to test the appearance of $Y_{\mathrm{e}}<0.5$ under such conditions, we increase the difference between the mean neutrino and antineutrino energies by hand. We evaluate expression (20) shown in Fig. 13b at $10 \mathrm{~km}$ outside the electron-neutrinosphere for 1.2 (dashed line) and 1.5 (dash-dotted line) times larger electron-antineutrino mean energies. For the first value, $Y_{\mathrm{e}}$ decreases but matter remains slightly proton-rich, where for the latter value matter becomes neutronrich. Indeed, the larger the difference between neutrino and antineutrino spectra are, the lower becomes the electron fraction in the wind. Note that the luminosities and electron-neutrino energies remained unmodified for this experiment. Such an increase of the energy difference between neutrinos and antineutrinos could perhaps be related to the uncertainty of the EoS for nuclear matter, which will be discussed in the following paragraph.

The assumed PNS radii in previous wind studies reach about $10 \mathrm{~km}$ shortly ( $\leq 1 \mathrm{~s}$ ) after the onset of the explosion. We define the radius of the PNS as the position of the electronneutrinosphere at the steep density gradient at the PNS surface. The approximated inner boundary of the physical domain in most wind models is close to but still inside this radius. The position of the neutrinospheres and the contraction of the PNSs found in the present paper differ significantly from the assumptions made in most previous wind studies. We find PNS radii of about $40 \mathrm{~km}$ at the time of the explosion and $20 \mathrm{~km}$ at about $2 \mathrm{~s}$ after bounce (see Fig. 15a). During the later evolution, the PNS contraction slows down. The PNS profile and hence the position of the neutrinospheres as well as the contraction behavior itself is given implicitly by the EoS for hot and dense nuclear matter as well as the PNS deleptonization. For the stiff EoS from Shen et al. (1998) and both the 10.8 and $18 \mathrm{M}_{\odot}$ progenitors, the PNSs reach radii of $14.5-15 \mathrm{~km}$ only at about $10 \mathrm{~s}$ after bounce (see Fig. 15). The larger radii of the neutrinospheres result in lower neutrino luminosities and mean energies and a lower difference between neutrino and antineutrino spectra in comparison to the assumptions made in most previous wind models. This is in agreement with Arcones et al. (2007) who additionally assume PNS radii of $15 \mathrm{~km}$ and find conditions that differ more from previous wind studies. They obtained significantly higher values for the electron fraction. To summarize, this effects and the different behavior of the neutrino spectra assumed in the previous wind studies leads to different matter properties of the neutrino-driven wind. A detailed comparison study of fast and slow contracting PNSs with respect to the neutrino-driven wind, e.g. applying EoSs with different compressibilities and asymmetry energies, would be necessary in the context of radiation hydrodynamics simulations using spectral Boltzmann neutrino transport. 
(a)

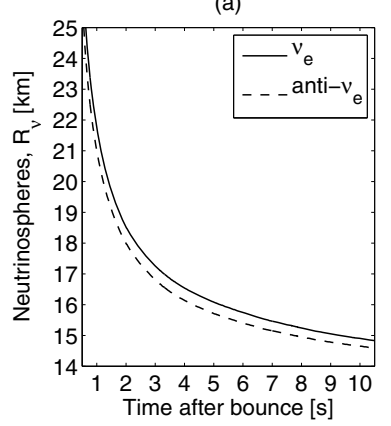

(b)

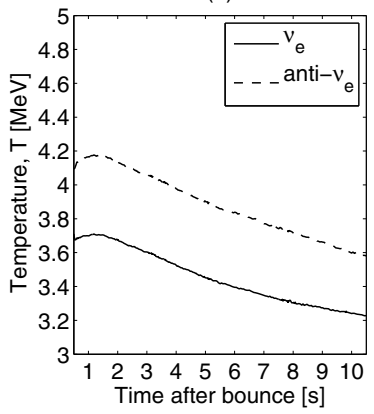

(c)

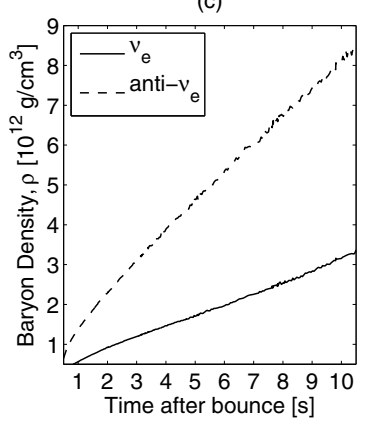

(d)

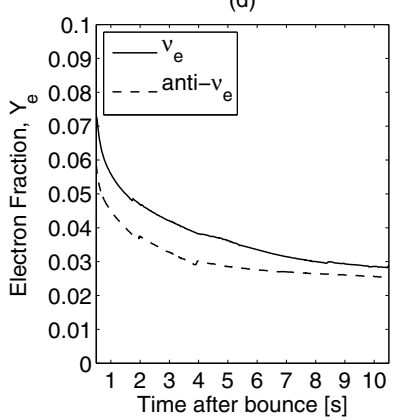

Fig. 15. Evolution of the neutrinosphere radii in graph a) and temperature, density and electron fraction at the corresponding neutrinospheres in graphs b), c) and d) respectively for the $10.8 \mathrm{M}_{\odot}$ progenitor model from Woosley et al. (2002).

(a)

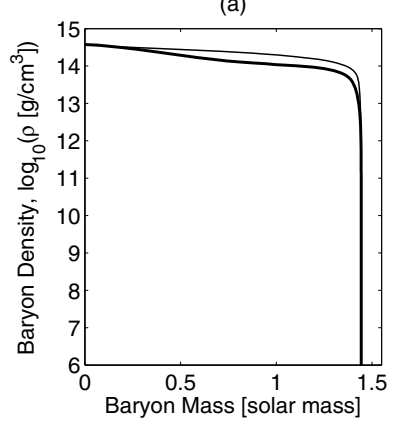

(b)

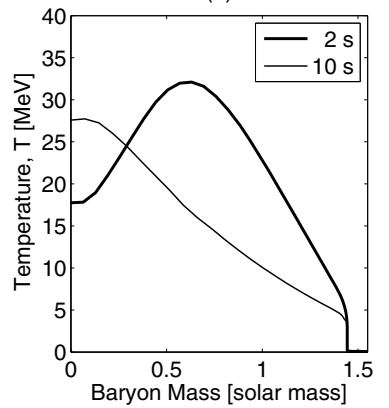

(c)

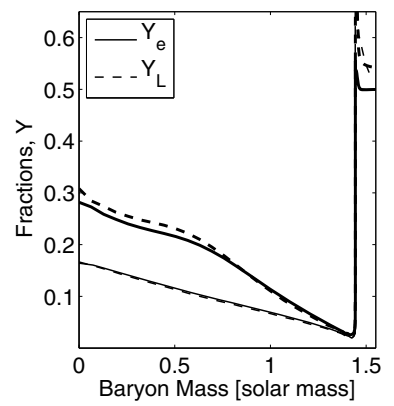

(d)

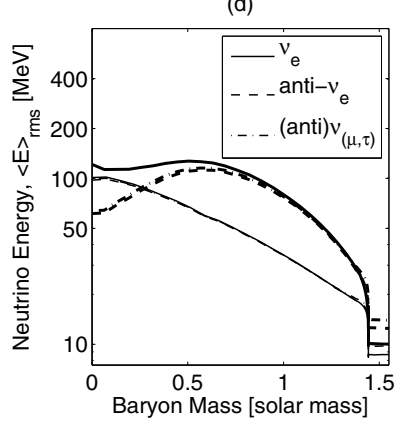

Fig. 16. Radial PNS profiles at two different post-bounce times (thick lines: $2 \mathrm{~s}$, thin lines: $10 \mathrm{~s}$ ), for the $10.8 \mathrm{M}_{\odot}$ progenitor model from Woosley et al. (2002).

\section{Long term post-bounce evolution}

During the neutrino-driven wind phase, the neutrino luminosities and mean neutrino energies decrease continuously, which leads to a constant decrease in the net-heating rates. At luminosities below $10^{51} \mathrm{erg} / \mathrm{s}$ (see Fig. 14), the supersonic matter outflow for the $10.8 \mathrm{M}_{\odot}$ progenitor model descends into a subsonic expansion. The wind termination shock turns again into a subsonic neutrino-driven wind. At later times, the neutrino-driven wind settles down to a quasi-stationary state with no significant matter outflow, illustrated at the example of the $18 \mathrm{M}_{\odot}$ progenitor model in Fig. 17a. The explosion shock continues to expand and the material enclosed inside the mass cut accretes onto the PNS at the center. In combination with the deleptonization, this leads to the continuous PNS contraction. However, the contraction proceeds on a timescale of seconds and hence the PNS can be considered in a quasi-stationary state. The dense and still hot and lepton-rich PNS at the center is surrounded by a low density and high entropy atmosphere, composed of light and heavy nuclei. See for example the abundances of the $18 \mathrm{M}_{\odot}$ progenitor for the post-bounce time of $22 \mathrm{~s}$ in Fig. 17e. The region at sub-saturation densities where light nuclei are present belongs to the inhomogeneous matter phase where clusters, known as pasta- and spaghetti-phases, are predicted to dominate the EoS. However, the EoS from Shen et al. (1998) approximates these effects by the presence of light nuclei represented in our model by $\alpha$-particles.

The internal temperature profile of the PNS is not constant. The central region of the PNS did not experience shock heating immediately after the Fe-core bounce, since the initial shock forms at the edge of the bouncing core. Its mass scales roughly with $Y_{\mathrm{e}}^{2}$ and is typically around values of $0.5-0.6 \mathrm{M}_{\odot}$ for lowand intermediate-mass $\mathrm{Fe}$-core progenitors. Hence, the central temperature after bounce is given by the thermodynamic conditions at bounce. The temperature changes only during the postbounce evolution due to compressional heating and the diffusion of neutrinos. The shock heated material inside the PNS shows significantly higher temperatures than at the center. The temperature decreases again towards the PNS surface where the matter is less dense (for the illustration of the radial temperature profile inside the PNS as well as the dynamical evolution of temperature and density, see Figs. $17 \mathrm{f}$ and $\mathrm{b}$ at selected postbounce times between 5-22 s). The neutrinos diffuse continuously out of the PNS and carry away energy. The central electron fraction reduces from $Y_{\mathrm{e}} \simeq 0.25$ at the onset of the explosion to $Y_{\mathrm{e}} \simeq 0.15$ at $22 \mathrm{~s}$ after bounce (see Fig. 17d). It relates to a temperature decrease from about $35 \mathrm{MeV}$ initially (at $3 \mathrm{~s}$ post-bounce) to $23 \mathrm{MeV}$ at about $22 \mathrm{~s}$ post-bounce. This corresponds to the initial and neutrino dominated cooling phase. Unfortunately the achieved temperatures are not representative since important neutrino reactions, such as the direct and modified Urca processes, are not yet taken into account.

\section{Discussion}

The neutrino-driven wind was found to occur in all three progenitor models under investigation, the $8.8 \mathrm{M}_{\odot} \mathrm{O}-\mathrm{Ne}-\mathrm{Mg}$-core and the 10.8 and $18 \mathrm{M}_{\odot} \mathrm{Fe}$-core progenitor models. Because the neutrino-driven explosions for the $\mathrm{Fe}$-core progenitors are launched using artificially enhanced neutrino reaction rates, one may ask about the impact of these modified rates to the neutrinodriven wind. Therefore we performed additional runs for which we switch back to the standard opacities given in Bruenn (1985) after the explosions have been launched. The times when we switch back is about $500 \mathrm{~ms}$ after bounce, chosen such that the dynamics of the explosion ejecta does not change anymore 
(a)

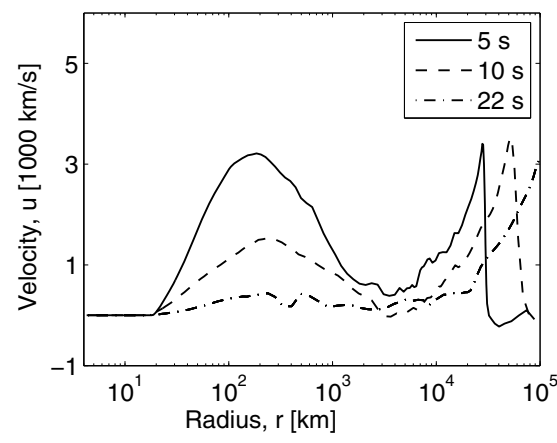

(c)

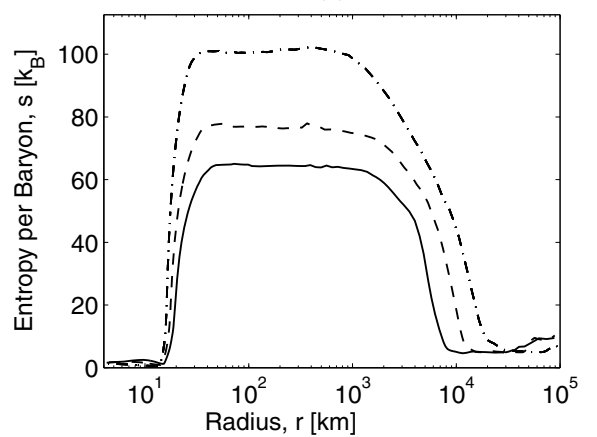

(e)

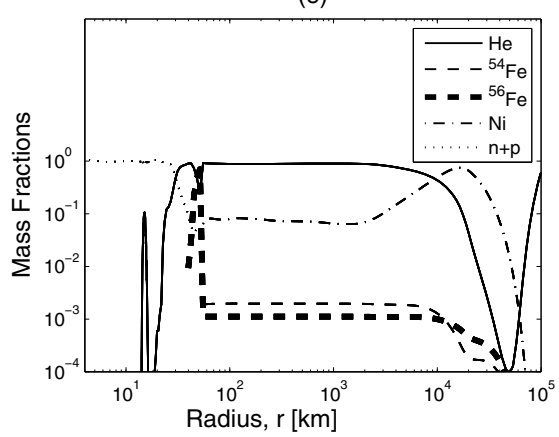

(b)

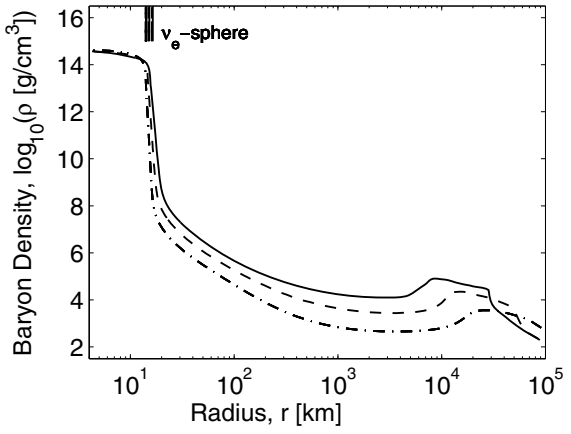

(d)

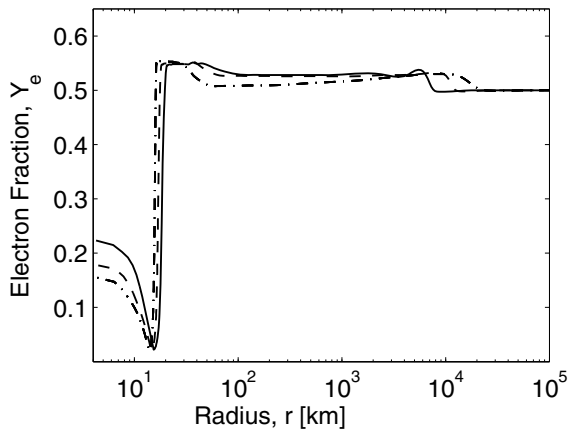

(f)

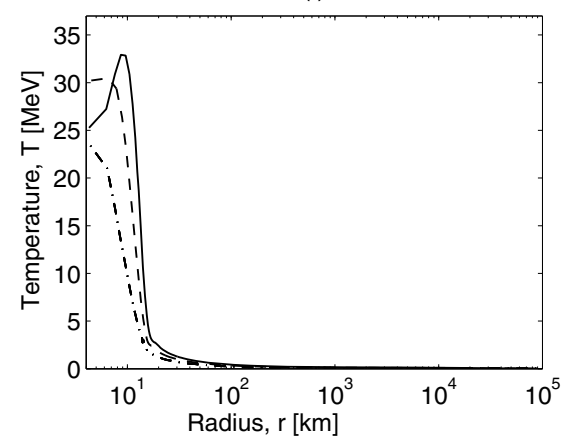

Fig. 17. Radial profiles of selected hydrodynamic variables for the $18 \mathrm{M}_{\odot}$ progenitor model at three different post-bounce times, illustrating the disappearance of the neutrino-driven wind and the PNS cooling and contraction. Graph e) illustrates the composition at $22 \mathrm{~s}$ post-bounce. significantly due to neutrino heating. However, the lower opacities translate to a significantly lower net-heating by a factor of 5-6 in the region on top of the PNS where the neutrino-driven wind develops. The energy deposition is still sufficient to drive the neutrino-driven wind but the matter velocities are lower by a factor of 2-5 in comparison to the wind velocities using the enhanced reaction rates (see Fig. 18a). The main effect of the artificially enhanced reaction rates and the hence increased neutrino heating to the dynamics is clearly the stronger neutrino-driven wind. For the $10.8 \mathrm{M}_{\odot}$ progenitor model and with the enhanced heating, the wind even develops supersonic velocities (as discussed above in Sect. 4) in Fig. 18a (top panel). The supersonic wind collides with the explosion ejecta where matter decelerates and hence the reverse shock forms, which additionally increases the entropy in the wind (see Fig. 18c, top panel). This additional entropy increase is absent in the simulations using the standard opacities, where the wind stays subsonic. This is also the case for the $18 \mathrm{M}_{\odot}$ progenitor model (Fig. 18, bottom panel), with and without the enhanced opacities. The neutrino-driven wind of the $\mathrm{O}-\mathrm{Ne}-\mathrm{Mg}$-core is illustrated in Fig. 11 using the standard rates based on Bruenn (1985). The formation of a supersonic neutrino-driven wind could be confirmed including the formation of the wind termination shock. Hence, one may speculate whether only low-mass progenitors develop strong neutrinodriven winds, while for more massive progenitors the influence of the winds to the matter properties of the ejecta becomes small. The progenitor dependency of the neutrino-driven wind is related to the density of the envelope surrounding the PNS after the explosion has been launched, which is significantly higher for more massive progenitors and hence the neutrino-driven wind is weaker.

The agreement of the time evolution of the mean neutrino energies between all three progenitor models under investigation (using the enhanced and standard opacities) in Fig. 14 is striking. The impact of the artificial heating to the neutrino observables and hence to the electron fraction in the wind is less pronounced. The influence on the composition of the wind is illustrated via the electron fraction in Fig. 18d. Using the standard rates, the wind stays slightly more proton-rich. Increasing the charged current reaction rates allows $\beta$-equilibrium to be established on a shorter timescale. In addition, matter stays slightly more protonrich for the less intense neutrino-driven wind, which develops for the Fe-core progenitors using the standard neutrino opacities. The additional electron fraction decrease in the neutrino-driven 
(a)

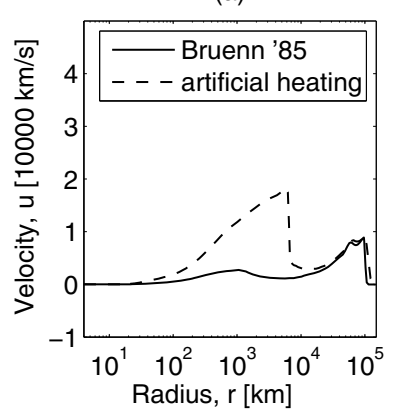

(a)

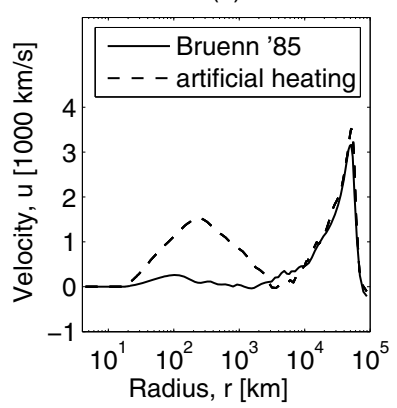

(b)

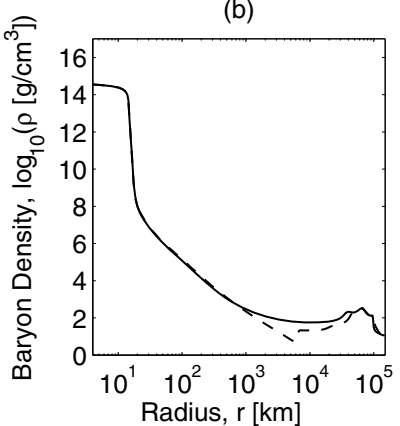

(b)

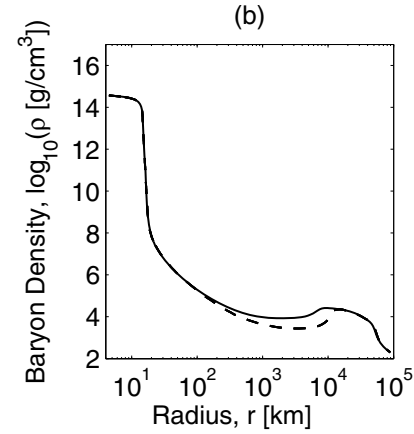

(c)

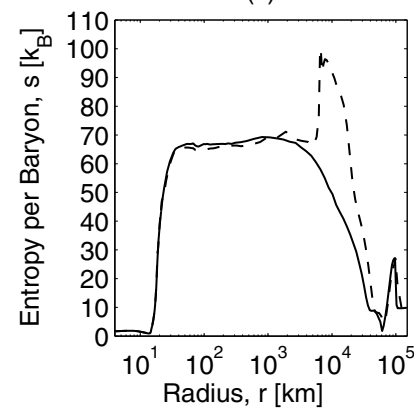

(c)

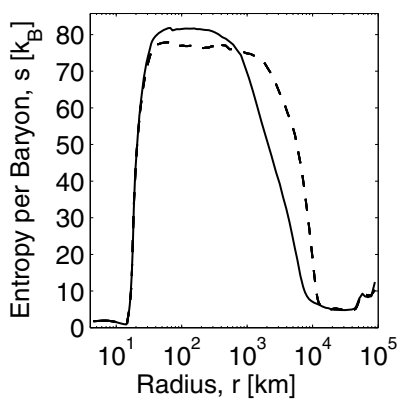

(d)

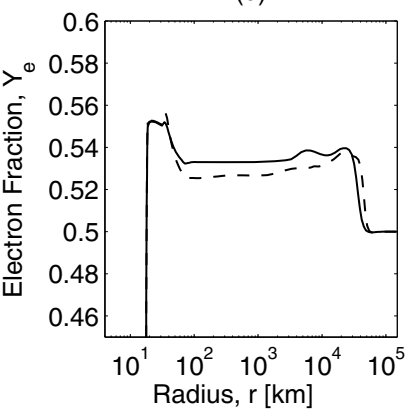

(d)

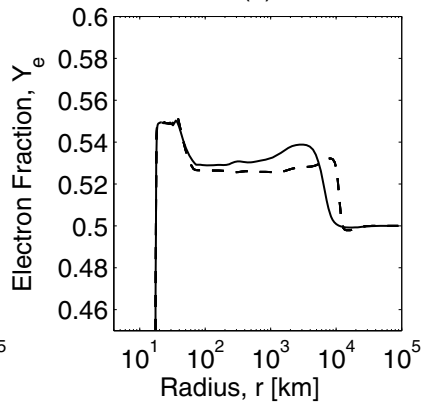

Fig. 18. Comparing selected hydrodynamic variables using the standard reactions rates based on Bruenn (1985) (solid lines) and the artificially enhanced rates (dashed lines) for the $10.8 \mathrm{M}_{\odot}$ (top) and the $18 \mathrm{M}_{\odot}$ progenitor model (bottom).

wind for the models using the enhanced neutrino reactions is found due to the higher degeneracy obtained in the stronger deceleration behind the explosion ejecta, and is therefore a dynamic effect. However, the findings of generally proton-rich ejecta as well as the generally proton-rich neutrino-driven wind does not change. The corresponding densities and entropies per baryon in the wind are shown in Figs. $18 \mathrm{~b}$ and c. The effects of the artificial heating are slightly lower entropies per baryon. The higher matter outflow velocities in the wind region using the artificial heating results additionally in lower densities, shown in Fig. 18b.

The artificially increased charged current reaction rates cannot be justified by physical uncertainties of the rates themselves. Similar to the high luminosities assumed in Arcones et al. (2007), they could be seen as a lowest order attempt to take the effects of multi-dimensional phenomena into account. For example, known fluid instabilities increase the neutrino energy deposition efficiency. Convection allows matter to stay for a longer time in the neutrino heating region (see Herant et al. 1994; Janka \& Müller 1996). Present axially symmetric core collapse models of massive Fe-core progenitor stars (even non-rotating) predict bipolar explosions (see Janka et al. 2008). The deviation from a spherical description and hence the deformation of the SAS due to fluid instabilities takes place during the neutrino heating phase on a timescale of several $100 \mathrm{~ms}$ after bounce. In multidimensional models, the luminosities are powered by a significantly higher mass accretion because the up-streaming neutrino heated matter is accompanied by large down-streams of cold material. These higher luminosities may power a strong (even supersonic) neutrino-driven wind behind the explosion ejecta, while the neutrino-driven wind may remain absent in the angular directions of the accreting material which will not be ejected.

Previous wind models have long been investigated as a possible site for the production of heavy elements via the $r$-process, motivated by the expectation of the ejection of neutron-rich material, the high entropies per baryon in the neutrino-driven wind and the short timescale of the neutrino-driven wind expansion (see Hoffman et al. 2007; Panov \& Janka 2009; and references therein). The relevant quantities are shown in Figs. 10 and 11 for the $10.8 \mathrm{M}_{\odot}$ and $8.8 \mathrm{M}_{\odot}$ progenitor models respectively. Illustrated are several selected mass shells that are part of the region where the neutrino-driven wind develops in our radiation hydrodynamics model based on spectral three-flavor Boltzmann neutrino transport. The inclusion of neutrino transport in a dynamical model is essential in order to obtain consistent neutrino spectra which determine the evolution of the electron fraction and the PNS contraction due to deleptonization and mass accretion. In comparison to previous static steadystate and dynamic wind models - where these ingredients were assumed - we confirm several properties of the accelerated material in the neutrino-driven wind, such as the fast expansion and the high matter outflow rate shown in Fig. 19, the high velocities in the Figs. $10 \mathrm{f}$ and $11 \mathrm{f}$ and the rapidly decreasing density and temperature of the accelerated material in Figs. 10b and c respectively. The expansion timescale in Fig. 19 is given by the following expression

$\tau_{\text {dyn }}=\left.\frac{r}{v}\right|_{T=0.5 \mathrm{MeV}}$,

evaluated at the surface of constant temperature of $T=0.5 \mathrm{MeV}$, compared with an alternative definition of the dynamic timescale which has been introduced in Thompson et al. (2001)

$\tau_{\rho}=\left|\frac{1}{v} \frac{1}{\rho} \frac{\partial \rho}{\partial r}\right|_{T=0.5 \mathrm{MeV}}^{-1}$,

as well as a timescale approximation which has been derived in Qian \& Woosley (1996) Eq. (61)

$\tau_{\mathrm{QW}} \propto \frac{1}{L_{\bar{v}_{\mathrm{e}}}} \frac{1}{\epsilon_{\bar{v}_{\mathrm{e}}}} R_{\mathrm{PNS}} M_{\mathrm{PNS}}$, 
T. Fischer et al.: protoneutron star evolution and the neutrino-driven wind
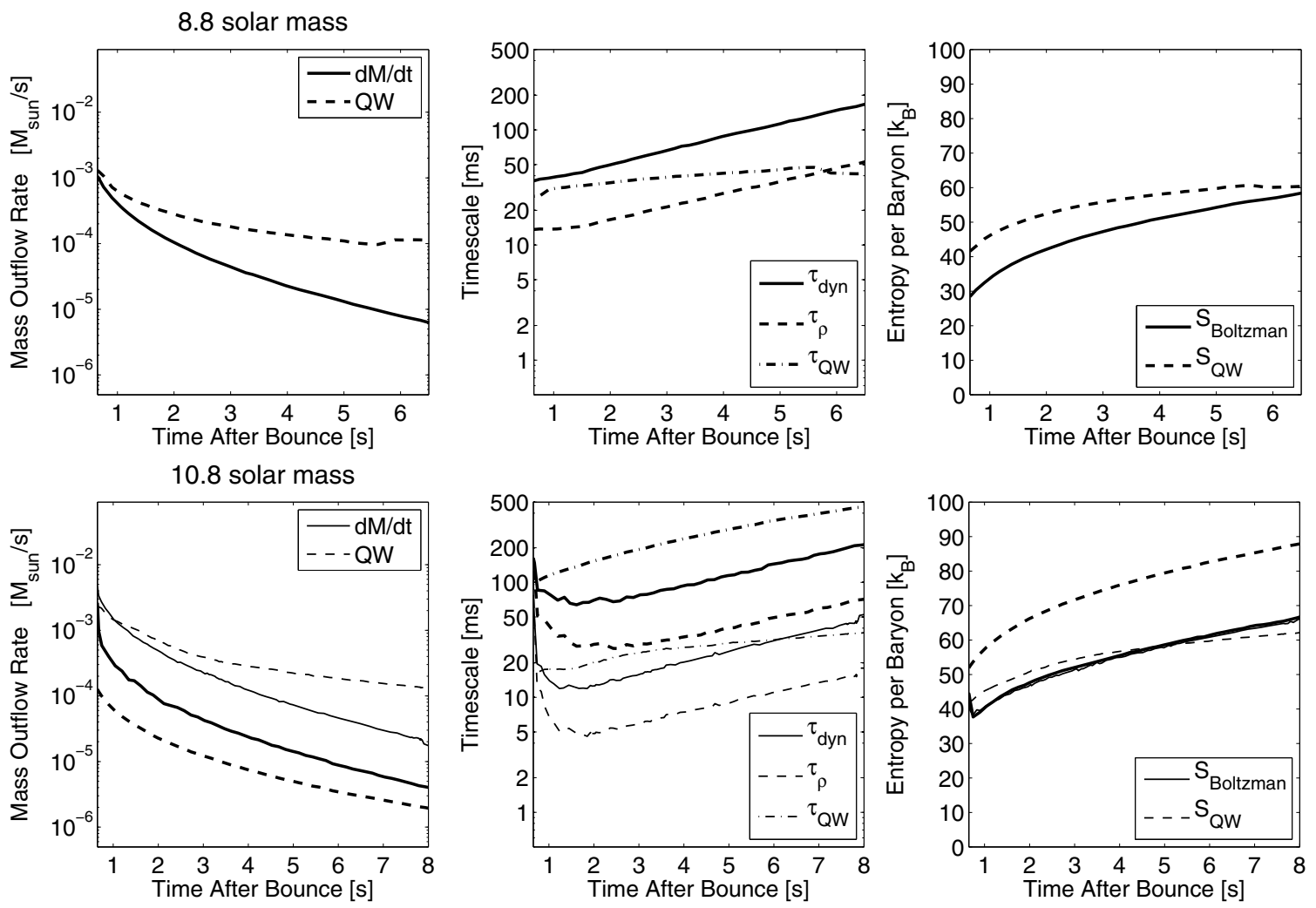

18 solar mass
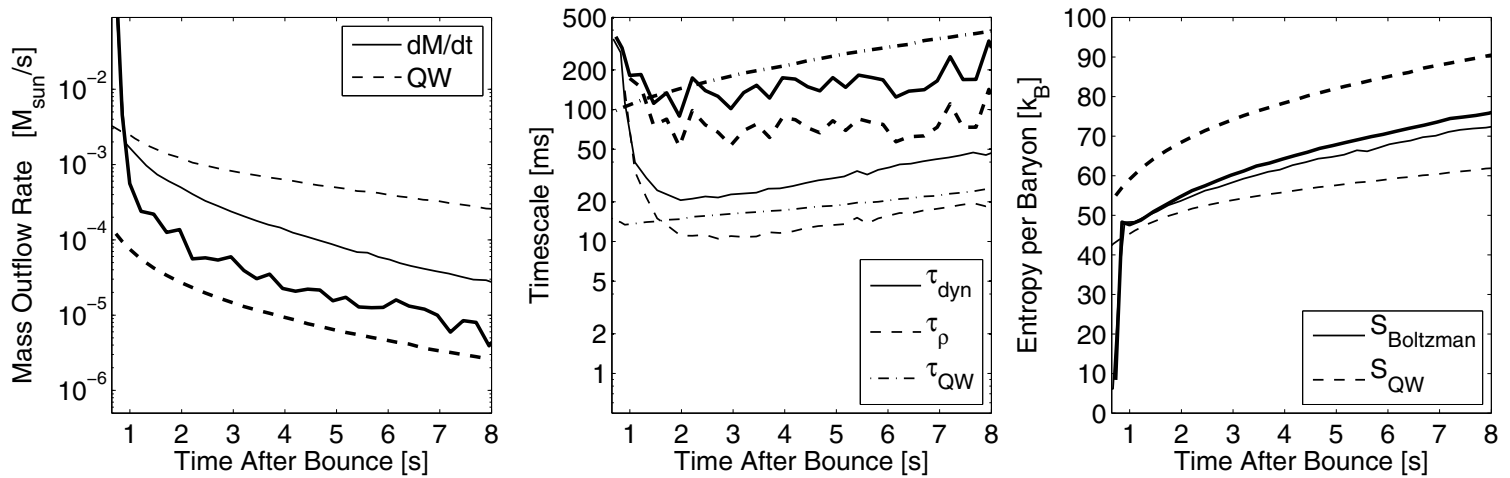

Fig. 19. Mass accretion rate, timescales and entropy in the wind for the three progenitor models under investigation, $8.8 \mathrm{M}_{\odot}$ (top), $10.8 \mathrm{M}_{\odot}$ (middle) $18 \mathrm{M}_{\odot}$ (bottom). The thick lines show data from simulations using the standard rates from Bruenn (1985), where a relatively weak neutrino-driven wind was obtained, and the thin lines show data from simulations using the enhanced rates.

where additionally approximations for the mass outflow rate and the entropy per baryon are derived as follows

$\left(\frac{\mathrm{d} M}{\mathrm{~d} t}\right)_{\mathrm{QW}} \propto L_{\bar{\gamma}_{\mathrm{e}}}^{5 / 3} \epsilon_{\bar{\gamma}_{\mathrm{e}}}^{10 / 3} R_{\mathrm{PNS}}^{5 / 3} M_{\mathrm{PNS}}^{-2}$,

$S_{\mathrm{QW}} \propto \frac{1}{L_{\bar{v}_{\mathrm{e}}}^{1 / 6}} \frac{1}{\epsilon_{\overline{\mathrm{v}}_{\mathrm{e}}}^{1 / 3}} \frac{1}{R_{\mathrm{PNS}}^{2 / 3}} M_{\mathrm{PNS}}$,

where $R_{\text {PNS }}$ and $M_{\text {PNS }}$ are the PNS radius and mass respectively, which we take to be given by the electron-antineutrinosphere. $\epsilon_{\bar{v}_{e}}$ is again the ratio of mean-square electron-antineutrino energy to average electron-antineutrino energy and $L_{\bar{v}_{\mathrm{e}}}$ is the electron-antineutrino luminosity, both taken at the neutrinosphere. In comparison with previous wind studies (see for example Fig. 4 of Arcones et al. 2007), we find generally a longer timescale of $\tau_{\text {dyn }}=10-50 \mathrm{~ms}$ shown in Fig. 19 (middle column) for the 10.8 (middle panel) and $18 \mathrm{M}_{\odot}$ (bottom panel) Fe-core progenitors using the enhanced opacities (thin lines) and $\tau_{\text {dyn }}=40-200 \mathrm{~ms}$ in Fig. 19 (middle column, top panel) for the $8.8 \mathrm{M}_{\odot} \mathrm{O}-\mathrm{Ne}-\mathrm{Mg}$-core using the standard rates based on Bruenn (1985). This corresponds to a mass outflow rate of $10^{-3}-10^{-4} \mathrm{M}_{\odot} \mathrm{s}^{-1}$ shown in Fig. 19 (left column) for the 10.8 (middle panel) and $18 \mathrm{M}_{\odot}$ (bottom panel) $\mathrm{Fe}$-core progenitors using the enhanced opacities (thin lines) and $10^{-3}-10^{-5} \mathrm{M}_{\odot} \mathrm{s}^{-1}$ in Fig. 19 (left column, top panel) for the $8.8 \mathrm{M}_{\odot} \mathrm{O}-\mathrm{Ne}-\mathrm{Mg}$-core using the standard rates. Figure 19 also compares the mass outflow rate, timescale and entropy per baryon with the approximations derived in Qian \& Woosley (1996), which are in qualitative agreement with our data obtained via Boltzmann neutrino transport. The differences for the mass outflow and the entropy at later times are maximally on the order of $2-5$ and relate most likely to the crucial assumptions made during the derivation of the 
above expressions, e.g. hydrostatic equilibrium, $R_{\mathrm{PNS}}=10 \mathrm{~km}$, $L_{v_{\mathrm{e}}} \simeq L_{\bar{v}_{\mathrm{e}}}, \epsilon_{\bar{v}_{\mathrm{e}}}=20 \mathrm{MeV}$, which differ significantly from our findings. Furthermore, since the 10.8 and $18 \mathrm{M}_{\odot}$ wind models are obtained using enhanced opacities, Fig. 19 compares additionally simulations using these tuned rates (thin lines) with data obtained using the standard rates (thick lines) where again the approximations from Qian \& Woosley (1996) are in qualitative agreement with our findings. The differences between simulations based on enhanced rates (thin lines) and standard rates (thick lines) in Fig. 19, i.e. higher mass outflow rates, shorter timescales and slightly lower entropies per baryon, are due to the stronger dynamic effect of the more pronounced wind in the models using the enhanced opacities.

However, the wind entropies of $40-100 k_{\mathrm{B}}$ found (initially driven due to neutrino heating and additionally due to the deceleration in the reverse shock) are significantly smaller than often assumed in the literature and the previously accelerated matter does not become neutron-rich as the neutrino-driven wind decelerates behind the explosion ejecta but stays slightly proton-rich where $Y_{\mathrm{e}}=0.51-0.54$ for more than $10 \mathrm{~s}$. This, in combination with the much slower PNS contraction illustrated via the neutrinospheres in Fig. 15 in comparison to static steady-state and dynamic wind models suggest that the assumptions made in previous wind studies should be carefully reconsidered. With respect to Woosley et al. (1994) (e.g. Fig. 3), we find generally smaller mean neutrino energies which decrease with respect to time after bounce. This results in a decreasing difference between the electron flavor neutrino mean energies, while in Woosley et al. (1994) this difference increases. This fact in combination with the different PNS properties found in Woosley et al. (1994), enabled a strong neutrino-driven wind where high entropies up to $400 k_{\mathrm{B}}$ /baryon and a low electron fraction of $Y_{\mathrm{e}} \simeq 0.35-0.45$ was obtained. These properties of the neutrino-driven wind differ quantitatively from our results, where lower entropies per baryon are obtained and matter stays proton-rich for more than $10 \mathrm{~s}$.

\section{Summary and outlook}

For the first time, spherically symmetric core collapse supernova simulations based on general relativistic radiation hydrodynamics and three-flavor Boltzmann neutrino transport are performed consistently for more than $20 \mathrm{~s}$. We follow the dynamical evolution of low- and intermediate-mass progenitors through the collapse, bounce, post-bounce, explosion and neutrino-driven wind phases. The explosions of Fe-core progenitors of 10.8 and $18 \mathrm{M}_{\odot}$ are modeled using artificially enhanced opacities, while the explosion of the $8.8 \mathrm{M}_{\odot} \mathrm{O}-\mathrm{Ne}-\mathrm{Mg}$-core is obtained using the standard opacities. For all models under investigation, we confirm the formation and illustrate the conditions for the appearance of the neutrino-driven wind during the dynamical evolution after the explosions have been launched. For the O-Ne-Mg-core and the $10.8 \mathrm{M}_{\odot} \mathrm{Fe}$-core progenitor models, the supersonic neutrinodriven wind collides with the slower expanding explosion ejecta where due to the deceleration the neutrino-driven wind termination shock appears. We discuss the impact of the reverse shock for several properties of the ejecta and find general agreement with Arcones et al. (2007).

The comparison with approximate and static steady-state as well as parametrized dynamic wind models leads to a discrepancy in the obtained physical properties of the neutrino-driven wind. Although the evolution of the hydrodynamic variables are in general agreement, we find smaller neutrino luminosities and a different behavior of the mean neutrino energies. In particular, the differences between the neutrino and antineutrino luminosities and mean neutrino energies are smaller. These differences reduce with time as the PNSs contract, which results in generally proton-rich neutrino-driven winds over more than $10 \mathrm{~s}$ for all our models. Hence, the suggestion that the physical conditions in the neutrino-driven wind could be favorable for the nucleosynthesis of heavy elements via the $r$-process could not be confirmed. For the accurate determination of the yields of the neutrino-driven wind, detailed nucleosynthesis analysis based on a large nuclear reaction network, taking the $r$-, $p$ - and $v p$ processes into account, is required. In order to further support the robustness, improvements of the input physics such as weak magnetism and nucleon-nucleon recoil (following e.g. Horowitz 2002), taking the presence of light and heavy clusters of nuclei into account as well as different EoSs with respect to different PNS contraction behaviors, should be considered. These may have a strong influence on the properties of the neutrino spectra at the neutrinospheres and may therefore modify some of the results found in the present study of the neutrino-driven wind.

Our simulations are carried out until the neutrino-driven wind settles down to a quasi-stationary state leading to the initial and neutrino dominated PNS cooling phase. There, the simulations have to be stopped because important neutrino cooling processes like the direct and modified URCA processes are not taken into account yet. However, a smooth connection to isolated neutron or protoneutron star cooling studies comes into reach for future work (Henderson \& Page 2007).

Acknowledgements. The authors would like to thank A. Arcones and G. Martínez-Pinedo for discussions and helpful comments. The project was funded by the Swiss National Science Foundation grant numbers PP002106627/1, PP00P2-124879 and 200020-122287. The authors are additionally supported by CompStar, a research networking program of the European Science Foundation, and the Scopes project funded by the Swiss National Science Foundation grant. No. IB7320-110996/1. A. Mezzacappa is supported at the Oak Ridge National Laboratory, which is managed by UT-Battelle, LLC for the US Department of Energy under contract DE-AC05-00OR22725.

\section{References}

Arcones, A., Janka, H.-Th., \& Scheck, L. 2007, A\&A, 467, 1227

Arcones, A., Martínez-Pinedo, G., O’Conner, E., et al. 2008, Phys. Rev. C, 78, 015806

Bethe, H. A., \& Wilson, J. R. 1985, ApJ, 295, 14

Bruenn, S. W. 1985, ApJS, 58, 771

Bruenn, S. W., Dirk, C. J., Mezzacappa, A., et al. 2006, J. Phys. Conf. Ser., 46, 393

Burrows, A., Hayes, J., \& Fryxell, B. A. 1995, ApJ, 450, 830

Duncan, R. C., Shapiro, S. L., \& Wasserman, I. 1986, ApJ, 309, 141

Fischer, T., Whitehouse, S. C., Mezzacappa, A., Thielemann, F.-K., \& Liebendörfer, M. 2009, A\&A, 499, 1

Fröhlich, C., Hauser, P., Liebendörfer, M., et al. 2006a, ApJ, 637, 415

Fröhlich, C., Hix, W. R., Martínez-Pinedo, G., et al. 2006b, New Astron. Rev., 50,496

Fröhlich, C., Martínez-Pinedo, G., Liebendörfer, M., et al. 2006c, Phys. Rev. Lett., 96 (14), 142502

Henderson, J. A., \& Page, D. 2007, Ap\&SS, 308, 513

Herant, M., Benz, W., Hix, W. R., Fryer, C. L., \& Colgate, S. A. 1994, ApJ, 435, 339

Hix, W. R., Messer, O. B. E., Mezzacappa, A., et al. 2003, Phys. Rev. Lett., 91, 201102

Hoffman, R. D., Woosley, S. E., \& Qian, Y. Z. 1997, ApJ, 482, 951

Hoffman, R. D., Pruet, J., Fisker, J. L., et al. 2007, unpublished [arXiv: 0712.2847]

Horowitz, C. J. 2002, Phys. Rev. D, 65, 043001

Janka, H.-Th. 2001, A\&A, 368, 527

Janka, H.-Th., \& Müller, E. 1995, ApJ, 448, L109 
T. Fischer et al.: protoneutron star evolution and the neutrino-driven wind

Janka, H.-Th., \& Müller, E. 1995, A\&A, 306, 167

Janka, H.-Th., Buras, R., Kitaura Joyanes, F. S., et al. 2005, Nuclear Physics A, 758,19

Janka, H.-Th., Marek, A., Müller, B., \& Scheck, L. 2008, In 40 Years of Pulsars: Millisecond Pulsars, Magnetars and More, ed. C. Bassa, Z. Wang, A. Cumming, \& V. M. Kaspi, AIP Conf. Ser., 983, 369

Kitaura, F. S., Janka, H.-Th., \& Hillebrandt, W. 2006, A\&A, 450, 345

Kotake, K., Sato, K., \& Takahashi, K. 2006, Rep. Prog. Phys., 69, 971

Langanke, K., Martínez-Pinedo, G., Sampaio, J. M., et al. 2003, Phys. Rev. Lett., 90, 241102

Liebendörfer, M. 2004, Proceedings of the 12th Workshop on Nuclear Astrophysics, Rep. No. MPA/P14, 143 [arXiv: astro-ph/0405029]

Liebendörfer, M., Mezzacappa, A., Thielemann, F.-K., et al. 2001a, Phys. Rev. D, 63, 103004

Liebendörfer, M., Mezzacappa, A., \& Thielemann, F.-K. 2001b, Phys. Rev. D, 63, 104003

Liebendörfer, M., Rosswog, S., \& Thielemann, F.-K. 2002, ApJS, 141, 229

Liebendörfer, M., Messer, O. E. B., Mezzacappa, A., et al. 2004, ApJS, 150, 263

Liebendörfer, M., Ramp, M., Janka, H.-Th., \& Mezzacappa, A. 2005, ApJ, 620, 840

Marek, A., \& Janka, H.-Th. 2009, ApJ, 694, 664

Mayle, R., \& Wilson, J. R. 1988, ApJ, 334, 909

Mezzacappa, A., \& Bruenn, S. W. 1993a, ApJ, 405, 637

Mezzacappa, A., \& Bruenn, S. W. 1993b, ApJ, 405, 669

Mezzacappa, A., \& Bruenn, S. W. 1993c, ApJ, 410, 740

Mezzacappa, A., \& Messer, O. E. B. 1999, J. Comput. Appl. Math., 109, 281

Mezzacappa, A., Blondin, J. M., Messer, O. E. B., \& Bruenn, S. W. 2006, In Origin of Matter and Evolution of Galaxies, AIP Conf. Ser., 847, 179
Miller, D. S., Wilson, J. R., \& Mayle, R. W. 1993, ApJ, 415, 278

Misner, C. W., \& Sharp, D. H. 1964, Phys. Rev., 136, 571

Nomoto, K. 1983, IAU Symp., 101, 139

Nomoto, K. 1984, ApJ, 277, 791

Nomoto, K. 1987, ApJ, 322, 206

Otsuki, K., Tagoshi, H., Kajino, T., \& Wanajo, S. Y. 2000, ApJ, 533, 424

Panov, I. V., \& Janka, H.-Th. 2009, A\&A, 494, 829

Qian, Y. Z., \& Woosley, S. E. 1996, ApJ, 471, 331

Sagert, I., Fischer, T., Hempel, M., et al. 2009, Phys. Rev. Lett., 102 (8), 081101

Scheck, L., Kifondis, K., Janka, H.-T., \& Müller, E. 2006, A\&A, 457, 963

Schinder, P. J., \& Shapiro, S. L. 1982, ApJS, 50, 23

Shen, H., Toki, H., Oyamatsu, K., \& Sumiyoshi, K. 1989, Prog. Theor. Phys., 100,1013

Takahashi, K., Witti, J., \& Janka, H.-Th. 1994, A\&A, 286, 857

Thielemann, F.-K., Brachwitz, F., Höflich, P., Martinez-Pinedo, G., \& Nomoto, K. 2004, New Astron. Rev., 48, 605

Thompson, T. A., \& Burrows, A. 2001, Nucl. Phys. A, 688, 377

Thompson, T. A., Burrows, A., \& Meyer, B. S. 2001, ApJ, 562, 887

Timmes, F. X., \& Arnett, D. 1999, ApJS, 125, 277

Wanajo, S. 2006a, ApJ, 647, 1323

Wanajo, S. 2006b, ApJ, 650, L79

Wilson, J. R., \& Mayle, W. R. 1993, Phys. Rep., 227, 97

Witti, J., Janka, H.-Th., \& Takahashi, K. 1994, A\&A, 286, 841

Woosley, S. E., \& Baron, E. 1992, ApJ, 391, 228

Woosley, S. E., Wilson, J. R., Mathews, G. J., Hoffman, R. D., \& Meyer, B. S. 1994, ApJ, 433, 229

Woosley, S. E., Heger, A., \& Weaver, T. A. 2002, Rev. Mod. Phys., 74, 1015

Yueh, W. R., \& Buchler, J. R. 1976, Ap\&SS, 41, 221 